\title{
Single-molecule imaging of cytoplasmic dynein in cellulo reveals the mechanism of motor activation and cargo capture
}

\author{
Nireekshit Addanki Tirumala ${ }^{1}$, Gregory Redpath ${ }^{2}$, Pritha Dolai ${ }^{3,5}$, Natasha Kapoor-Kaushik ${ }^{4}$, Nicholas Ariotti $^{4}, \mathrm{~K}_{\mathrm{ijjay}}$ \\ Kumar $^{3}$, and Vaishnavi Ananthanarayanan ${ }^{2 凶}$ \\ ${ }^{1}$ Centre for BioSystems Science and Engineering, Indian Institute of Science, Bengaluru, India \\ ${ }^{2}$ EMBL Australia Node in Single Molecule Science, School of Medical Sciences, Sydney, University of New South Wales, Australia \\ ${ }^{3}$ International Centre for Theoretical Sciences, Tata Institute of Fundamental Research, Bengaluru, India \\ ${ }^{4}$ Electron Microscopy Unit, University of New South Wales, Sydney, Australia \\ ${ }^{5}$ Current affiliation: Instituut voor Theoretische Fysica, Katholieke Universiteit Leuven, Belgium
}

\begin{abstract}
Cytoplasmic dynein 1 (dynein) is the primary minus end- 43 directed motor protein in most eukaryotic cells. Dynein remains 44 in an inactive conformation until the formation of a tripartite ${ }_{45}$ complex comprising dynein, its regulator dynactin and a cargo ${ }_{46}$ adaptor. How this process of dynein activation occurs is unclear, since it entails the formation of a three-protein complex inside the crowded environs of a cell. Here, we employed livecell, single-molecule imaging to visualise and track fluorescently tagged dynein. First, we observed that only $\sim \mathbf{3 0 \%}$ of dynein ${ }^{50}$ molecules that bound to the microtubule (MT) engaged in mi- 51 nus end-directed movement, and that too for a short duration of 52 $\sim 0.6 \mathrm{~s}$. Next, using high-resolution imaging in live and fixed cells, 53 and using correlative light and electron microscopy, we discov- 54 ered that dynactin remained persistently attached to MTs, and ${ }_{55}$ endosomal cargo remained in proximity to the MTs and dynactin. Finally, we employed two-colour imaging to visualise cargo movement effected by single motor binding. We then used these discoveries as the basis for a stochastic model incorporating dynamic motors binding to cargo located along MTs, and also developed a coarse-grained 3-state run-and-tumble particle (RTP) model for the cargo that quantitatively recapitulates ${ }^{6}$ the emergent statistics of cargo movement. Taken together, we 62 discovered a search mechanism that is facilitated by dynein's 63 frequent MT binding-unbinding kinetics: (1) in a futile event 64 when dynein does not encounter cargo anchored in proximity to 65 the MT, dynein dissociates and diffuses into the cytoplasm, (2) 66 when dynein encounters cargo and dynactin upon MT-binding, it moves cargo in a short run. Several of these short runs are undertaken in succession for long-range directed movement. In conclusion, we demonstrate that dynein activation and cargo capture are coupled in a step that relies on the reduction of ${ }^{70}$ dimensionality to enable minus end-directed transport in $\mathrm{cel}^{-7}$ lulo, and that complex cargo behaviour emerges from stochastic ${ }^{72}$ motor-cargo interactions.
\end{abstract}

cytoplasmic dynein | dynactin | cargo trafficking | single-molecule imaging | stochastic transport

Correspondence: vaish@unsw.edu.au

\section{Introduction}

minus-end directed movement of a variety of cargo in cells containing MTs (2). Dynein is a large complex of homodimers comprising 500kDa heavy chains, and other accessory proteins including light chains, light intermediate chains and intermediate chains, which mediate dimerisation of dynein and thereby its processivity.

The activity of motor proteins is typically regulated: several kinesins assume an autoinhibited conformation until attachment to cargo (3); dynein was first found to be regulated for its processivity by the multisubunit complex, dynactin (4). More recent studies have additionally implicated cargo adaptors - which link dynein to a multitude of cargo - in the activation of dynein (5-8). Dynactin is a large multi subunit complex (9) that was first identified as an activator of minus-end directed motility of vesicles (10). Further research indicated that an intact dynactin complex was necessary for dynein's function and that dynactin could interact with MTs via its p150 subunit $(11,12)$. The N-terminal CAP-Gly domain on p150 was also found to be able to interact with growing MT plus ends via EB1/CLIP-170 pathway $(13,14)$ and influence intracellular transport (15). However, the interaction between dynein and dynactin, as probed from co-immunoprecipitation assays, was observed to be weak, and over expressing the $\mathrm{N}$ terminal (cytoplasmic) fragment of the cargo adaptor BiCD2 was found to significantly increase dynein-dynactin interaction (16).

Cargo adaptors are proteins with transmembrane and cytoplasmic domains that link membranous cargo to the motor (17), with specific cargo adaptors being unique to different types of cargo $(18,19)$. Recent single molecule in vitro research has established that formation of the dynein-dynactincargo adaptor (DDC) complex is essential for processive motion $(20,21)$. Cryo-EM studies later revealed that the formation of the DDC complex relieved the autoinhibition of dynein, and reoriented the motor for processive movement (22-24). The DDC complex ameliorates the force produced by single dynein motors from $1 \mathrm{pN}$ to about $6 \mathrm{pN}$ (25). Thus our current understanding suggests that formation of DDC complex is essential in dynein driven transport.

While it is clear that the tripartite complex formation is an essential first step in the activation of the dynein motor, 

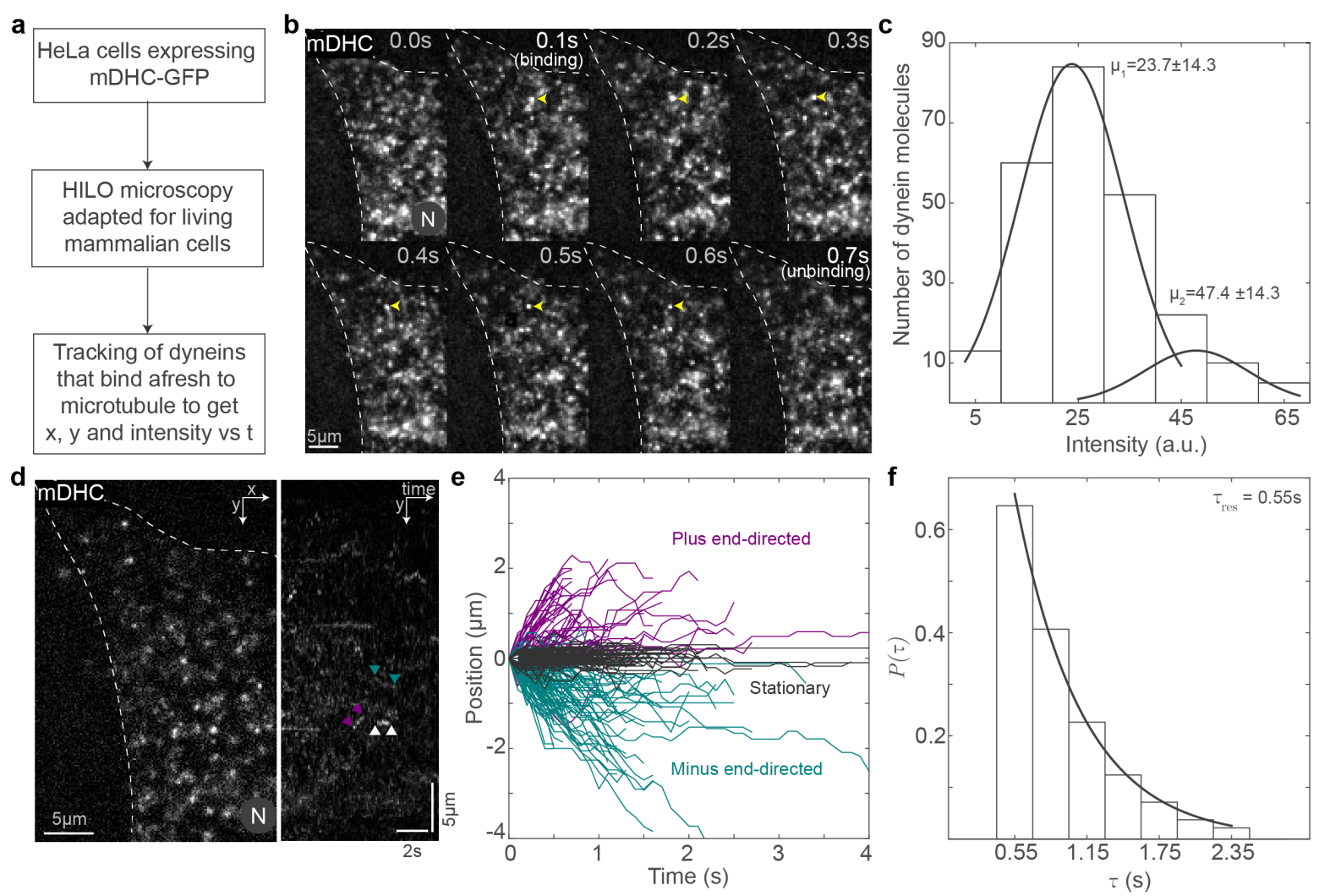

Fig. 1. Visualisation of single molecules of dynein in living cells. a, Schematic of the protocol followed for the visualisation of single molecules of dynein. $\mathbf{b}$, Montage of HILO images showing representative binding and unbinding events of a single fluorescent mDHC molecule ('(binding)' and '(unbinding)'). The single molecule is indicated with yellow arrowheads for the duration of the time it remains bound in the field of view. Time is indicated at the top right of each image in the montage. c, Intensity histogram of single molecules of dynein with the Gaussian fits (grey line). The mean \pm s.d. of the Gaussian distributions is indicated above the fits. d, HILO image (left) and kymograph (right) of a cell expressing mDHC-GFP. Representative stationary, minus end-directed and plus end-directed events are indicated with the white, teal and magenta arrowheads respectively in the kymograph. e, Plot of position vs. time for the single-molecule events tracked, showing stationary events (grey), minus end-directed events (teal) and plus end-directed events (magenta). $\mathbf{f}$, Histogram with the residence time of dynein on the MT on the $\mathrm{x}$ axis and $P(\tau)=1$-cumulative frequency on the $\mathrm{y}$ axis. The exponential fit (grey line) gave a mean residence time $\tau_{\text {res }}=1 / r_{\text {off }}^{D} \sim 0.55 \mathrm{~s}$. In $\mathbf{b}$ and d, 'N' marks the location/direction of the nucleus.

it is unknown how this process occurs in a living cell, amid 107 its crowded environs and the independent dynamics of each 108 component of the tripartite complex. Here, we employ sev- 109 eral strategies including single-molecule imaging, correlative 110 light and electron microscopy (CLEM), high-resolution fluorescence microscopy and stochastic modelling to reveal the mechanism of formation of the tripartite complex and therefore, activation of dynein.

\section{Results and Discussion}

Dynein interacts transiently with the MT. To visualise ${ }^{117}$ the behaviour of dynein in HeLa cells expressing mouse ${ }^{118}$ DYNH1C1-GFP (mDHC-GFP, (26)), we adapted and opti- ${ }^{119}$ mised highly inclined and laminated optical sheet (HILO) ${ }^{120}$ microscopy ((27), Fig. S1a). When cells expressing low lev- ${ }^{121}$ els of mDHC-GFP were observed under a spinning disk con- ${ }^{122}$ focal (SD) microscope, the fluorescence signal appeared cy- ${ }^{123}$ tosolic, with no discernible dynein punctae (Fig. S1b). How- ${ }^{124}$ ever, when the same cells were observed using our modified ${ }_{125}$ HILO microscopy, distinct fluorescent spots were visible. $\quad{ }_{126}$

We adapted our microscopy protocol to obscure dynein ${ }_{127}$ diffusing in the cytoplasm and to only observe dynein that ${ }_{128}$ was bound to and resided on the MT $((28,29)$, Fig. 1a, see 129 Methods). We observed that dynein spots appeared afresh 130 and remained in the field of imaging for a short duration (Fig. $1 \mathrm{~b}$, Video S1). We intuited that these corresponded to events where single dynein molecules previously diffusing in the cytoplasm bound to MTs (Fig. S1c-e).

To confirm that the appearance of fluorescent signal on the MT corresponded to binding of a single molecule of dynein to the MT, we analysed the intensity of these fluorescent spots (Fig.1c). For single dynein molecules, we would expect the intensity histogram to fit to a sum of two Gaussian distributions, one corresponding to a GFP fluorescing from one DHC and the other corresponding to two GFPs fluorescing from both DHCs in the genetic background of these cells. The former primarily arose due to photobleaching of GFP during the course of imaging. Accordingly, the intensity histogram of these fluorescent spots revealed that we indeed observed single dynein molecules since the intensity histogram fit best to a sum of two Gaussians, with the mean of the first Gaussian profile being half that of the second (Fig. 1c).

Next, we analysed the two-dimensional position ( $x$ and $y$ ) versus time $(t)$ of single molecules of dynein that bound afresh from the cytoplasm to the MT (Fig. 1d). Based on automated thresholding (see Methods), we classified the tracks as stationary, minus end-directed and plus end-directed. The plus ends of the MT were predominantly at the periphery in 
a
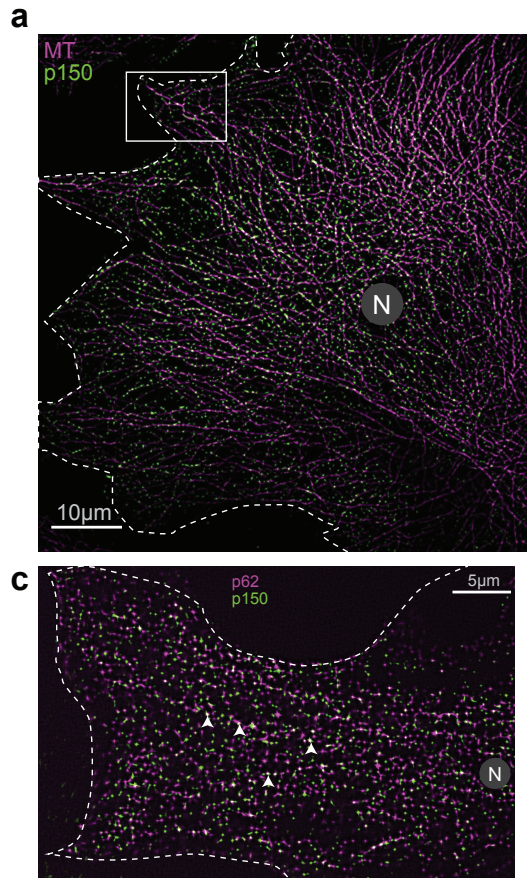

b
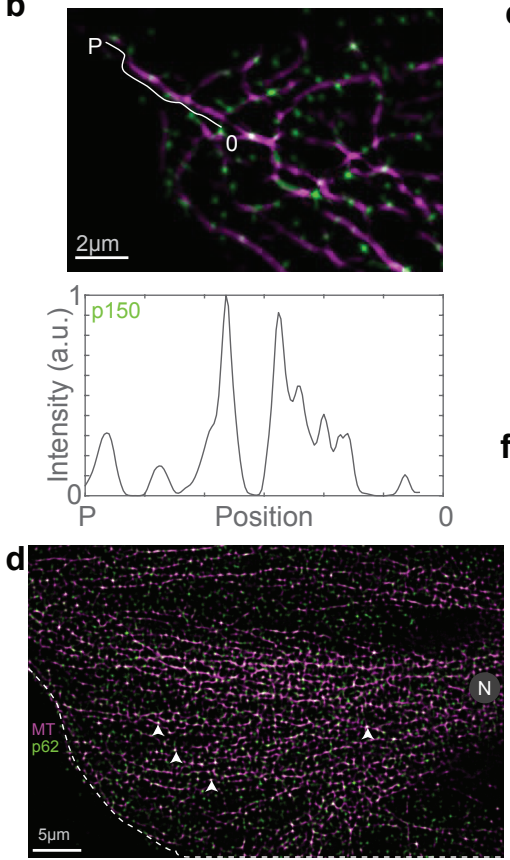

e

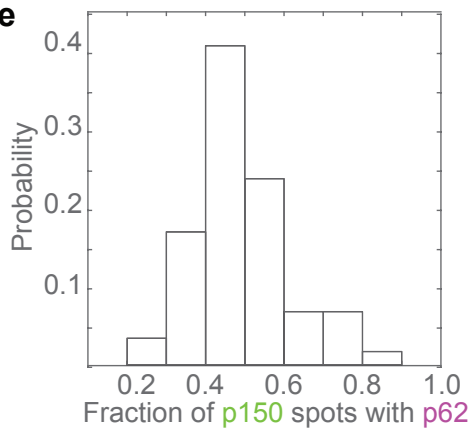

f

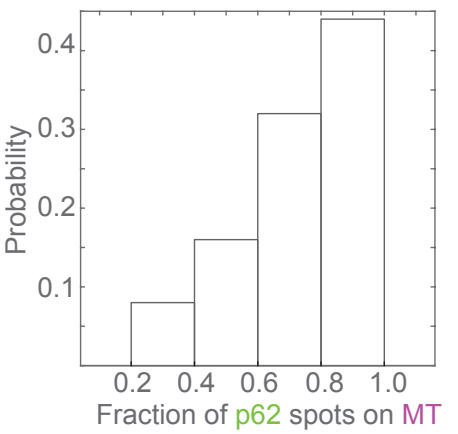

Fig. 2. The dynactin complex binds along the entire length of the MT. a, Immunofluorescence image of MT (magenta) and p150 (green) obtained using SD microscopy + SRRF. b, Enlarged view of the area marked with the white rectangle in a and the line profile of p150 intensity along the length of a representative MT from the plus end ('P') to $\sim 6 \mu \mathrm{m}$ from the plus end of the MT ('0'). c, Immunofluorescence image of p62 (green) and p150 (magenta) obtained using SD microscopy + SRRF. The white arrowheads indicate representative p150 spots that also contain p62. d, Immunofluorescence image of MT (magenta) and p62 (green) obtained using SD microscopy + SRRF. The white arrowheads indicate representative p62 spots that occur on the MT. e, Histogram of the probability of cooccurrence of p62 with p150, indicating a high likelihood of presence of the entire complex at a p150 spot. $\mathbf{f}$, Histogram of the probability of cooccurrence of p62 on the MT, which points to a high likelihood for the presence of the entire dynactin complex on the MT. In a, c and d, 'N' marks the location/direction of the nucleus.

these elongated cells (Fig. S1f), and hence we annotated 159 movement towards the cell center as minus end-directed, 160 and movement away as plus end-directed. We observed that ${ }_{161}$ $\sim 50 \%$ of all the dynein molecules tracked $\left(n=177 / 329, N=3_{162}\right.$ independent experiments from $>50$ cells) remained stationary ${ }_{163}$ upon MT binding, while $\sim 30 \%$ ( $n=95 / 329)$ moved towards ${ }_{164}$ the minus end (Fig. 1e). The remaining $20 \%$ moved to- 165 wards the plus end and these arose likely due to attachment ${ }_{166}$ of dynein to cargo being moved to the plus end by kinesins ${ }_{167}$ (Fig. 1e). The velocity measured for minus-end directed ${ }_{168}$ movement of single dyneins was $1.2 \pm 0.7 \mu \mathrm{m} / \mathrm{s}$ (mean \pm s.d.), ${ }_{169}$ similar to values reported for mammalian dynein previously 170 $(30,31)$. We also confirmed that the underlying MT was sta- 171 ble, did not undergo sliding, and therefore did not contribute ${ }_{172}$ to the dynein behaviour we observed (Fig. S1g).

We then measured the mean residence time $\left(\tau_{\text {res }}\right)$ of ${ }^{174}$ dynein on the MTs to be $0.55 \mathrm{~s}$ (95\% confidence interval (CI): 0.51-0.59 s) (Fig. 1f). The unbinding rate of dynein from MT 175 $r_{\text {off }}^{D}=1 / \tau_{\text {res }}$ was $1.7 \mathrm{~s}^{-1}$, which is similar to the previously 176 reported unbinding rate of single dyneins (32). We verified 177 that this short residence time of dynein on MTs was a true ${ }_{178}$ representation of the duration of time that dynein remained 179 attached to the MT and not convolved by GFP's photobleach- 180 ing time (Fig. S1h and i). Further, by knocking down endoge- 181 nous HeLa DYNC1H1 (hDHC), we verified that our obser- 182 vations were not an artefact of expression of mDHC-GFP in 183 this background (Fig. S2a-h). To the best of our knowledge, 184 these are the first observations of single molecules of dynein 185 in mammalian cells and indicate that dynein likely exists in an inactive state inside the cell, similar to reports from in vitro studies $(16,20,21,23)$.

The short residence time of dynein that we observed is in contrast with the findings from previous research $(20,21)$ which report a run length of upto $8 \mu \mathrm{m}$ for dynein. However, other in vitro results have reported dynein run lengths that are comparable with our results $(4,33)$. It is possible that longer run lengths were observed when dynein formed the DDC complex in vitro due to the use of the $\mathrm{N}$-terminal fragment of BiCD2 (BiCD2-N), as opposed to the autoinhibited, full length BiCD2. The former binds more strongly to dynein-dynactin. Moreover, the molar excess of adaptors used, the ionic strength of the buffers, and the source and modifications on the MTs all play a role in motor run length and, therefore, are not directly comparable to in vivo results.

\section{Dynactin remains persistently associated with MTs.}

Next, we aimed to visualise the dynamics of dynactin, the second player in the tripartite complex. Dynactin was first identified as a complex that was required for dynein-driven motility of vesicles in vitro (10). Several recent pieces of research have identified dynactin as an essential part of the active dynein complex $(4,24,34)$. Dynactin is a multi-subunit complex which binds to MTs independently of dynein via its $\mathrm{N}$-terminal p150 subunit (9). However, dynactin is unlikely to interact with dynein in the absence of the cargo adaptor $(16,20,21,23)$. 
a

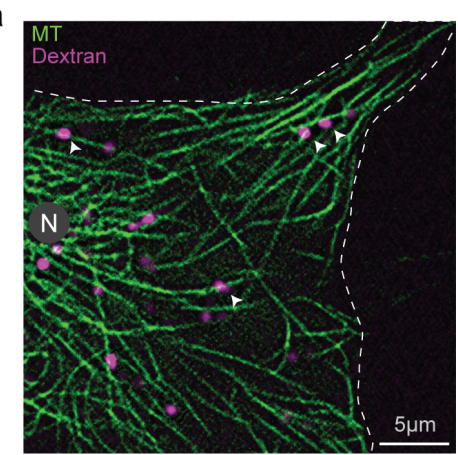

d

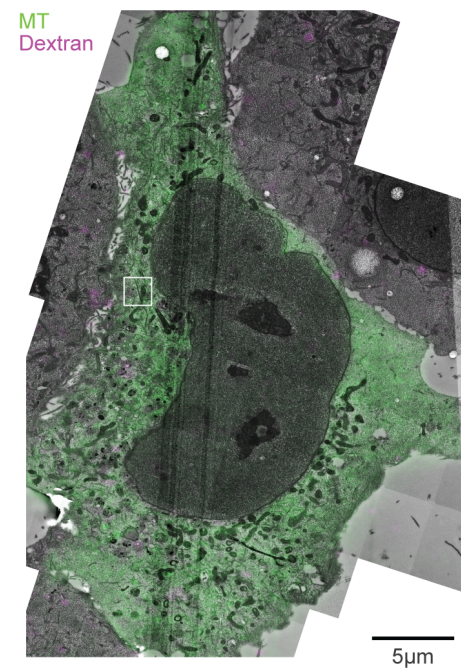

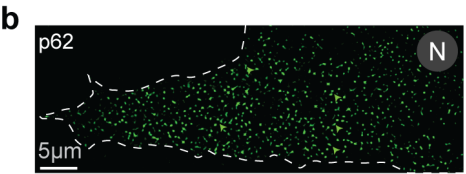
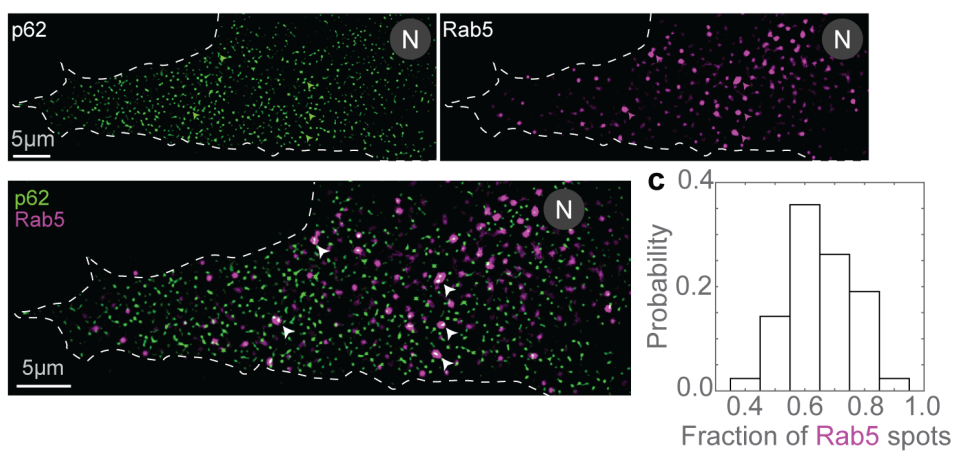
with 062
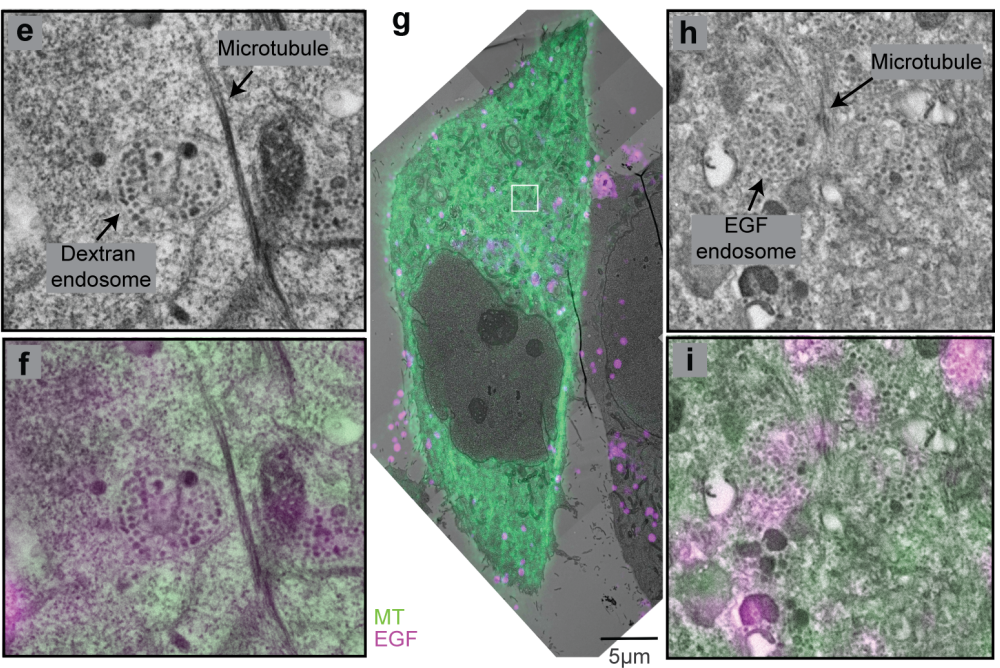

Fig. 3. Endosomes remain close to MTs. a, SD + SRRF image of MTs (green) and dextran vesicles (magenta) in live cells. The white arrowheads indicate representative vesicles on the MT. b, SD + SRRF image of p62 (top left), dextran vesicles (top right) in live cells and their merge (bottom, p62 in green and dextran in magenta). The white arrowheads in the merged image indicate representative vesicles that colocalise with p62, and are shown as green and magenta arrowheads in the p62 and dextran images respectively. c, Histogram of the probability of cooccurrence of p62 with Rab5, indicating a high likelihood of dynactin being present in a complex with endosomal cargo. d, Overlay of confocal images of MT (green) and dextran vesicles (magenta), and EM images of the same cell (grey). e, EM image of the region indicated with the white square in $\mathbf{d}$, showing a representative MT and dextran endosome. $\mathbf{f}$, Confocal fluorescence image of MTs (green) and dextran (magenta) of the region in e. $\mathbf{g}$, Overlay of confocal images of MT (green) and EGF vesicles (magenta), and EM images of the same cell (grey). $\mathbf{h}$, EM image of the region indicated with the white square in $\mathbf{g}$, showing a representative MT and EGF endosome. i, Confocal fluorescence image of MTs (green) and EGF (magenta) of the region in $\mathbf{h}$. In $\mathbf{a}$ and $\mathbf{b}$, 'N' marks the location/direction of the nucleus.

The +TIP protein EB1 has been found to recruit an- 207 other + TIP protein, CLIP-170, which in turn binds and clus- 208 ters dynactin via its p150 subunit at growing MT plus ends 209 (14). MT plus ends thus decorated with dynactin also ac- 210 cumulated dynein at these sites, and evidence suggested that ${ }_{211}$ cargo transport was initiated when these MT plus ends con- 212 tacted intracellular cargo $(15,35)$. However, MT plus end- 213 mediated initiation of dynein-driven transport appears to vary 214 with cell type and context $(14,36,37)$. Therefore, using SD 215 microscopy in combination with super-resolution radial fluc- 216 tuations (SRRF, (38)) we first quantified the localisation of ${ }_{217}$ p150 (Fig. 2a). Our high-resolution images revealed that ${ }_{218}$ only $\sim 17 \%$ of the MT plus ends were enriched with p150, and ${ }_{219}$ p150 appeared bound along the entire length of the MT lattice 220 (Fig. 2b). Further, by quantifying the intensities of mDHC- 221 GFP expressed in our cells, we concluded that the significant 222 MT plus end localisation of dynein reported in earlier studies 223 $(16,39)$ may represent an artefact of dynein overexpression 224 (Fig. S3a, b).

The dynactin complex, however, has been observed to ${ }^{226}$ show no MT-binding in the absence of dynein in in vitro as- ${ }^{227}$ says (20). To ascertain that the p150 spots we observed in these cells represented the entire dynactin complex, we used SRRF to visualise $\mathrm{p} 150$ in concert with another dynactin subunit, p62 (Fig. 2c). The p62 subunit of dynactin is located in the pointed end complex of dynactin (9), and colocalisation of p62 with p150 would indicate the presence of the complete dynactin complex. We observed that $49 \pm 12 \%$ (mean \pm s.d.) of the p150 spots colocalised with p62 $(n=21,934 / 44,306$ spots from $\mathrm{N}=2$ independent experiments with 59 cells, Fig. 2d). Additionally, we used SRRF to visualise the localisation of p62 on MTs. The presence of p62 on MTs would indicate association with the MT of a subunit which does not normally do so unless it is part of the entire dynactin complex. Therefore, occurrence of p62 on the MT would imply localisation of the entire complex on the MT via p150. We observed that $74 \pm 18 \%$ (mean \pm s.d.) of the p62 spots ( $\mathrm{n}=59,715 / 79,639$ spots from 1 experiment with 25 cells) were present on MTs (Fig. 2e, f). Therefore, the complete dynactin complex is likely present along the entire length of the MT lattice. Additionally, fluorescently tagged p150 had residence times on the MT that far exceeded that of dynein, with most spots of 

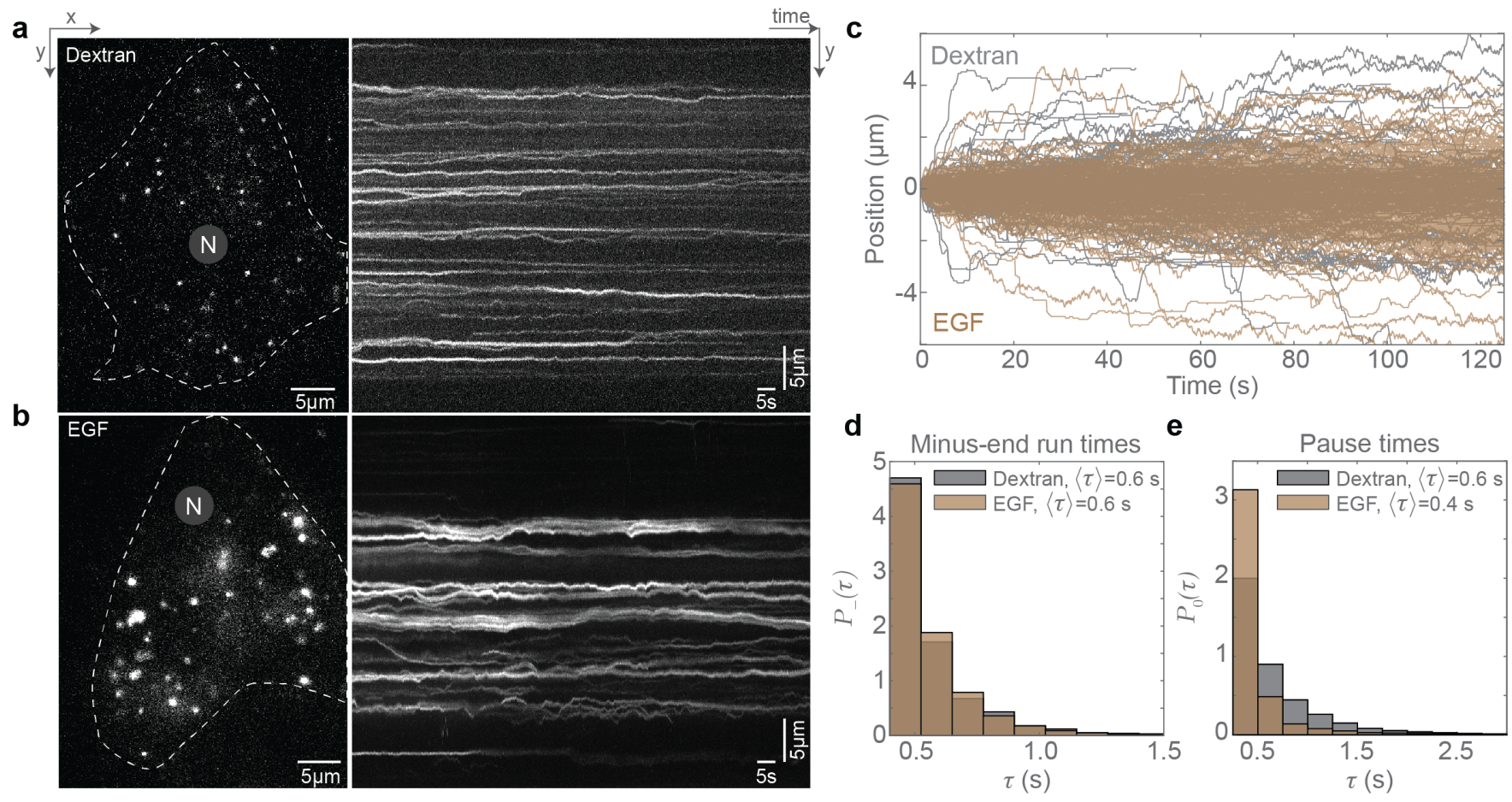

Fig. 4. Dextran and EGF vesicles display distinct movement kinetics. a, HILO image from a time-lapse of dextran vesicles in HeLa cells (left) and the corresponding kymograph (right). b, HILO image from a time-lapse of EGF vesicles in HeLa cells (left) and the corresponding kymograph (right). c, Plot of position versus time of dextran vesicles (grey) and EGF (brown) in HeLa cells. d, Probability distribution $P_{-}(\tau)$ of the minus end-directed run times for dextran (grey) and EGF (brown) vesicles. e, Probability distribution $P_{0}(\tau)$ of the pause times for dextran (grey) and EGF (brown) vesicles. In a and $\mathbf{b}$, 'N' marks the location/direction of the nucleus. In d and e, the average run/pause time $(\langle\tau\rangle)$ for dextran and EGF vesicles are indicated.

p150 remaining bound to the MT for the entire duration of ${ }_{257}$ the time-lapse video ( 30 s) (Fig. S3c, Video S2). Further, 258 we observed that depletion of p150 through siRNA mediated 259 silencing reduced the levels of both p150 and p62 along the 260 MT lattice (Fig. S3d-i). To test that the perturbation of dy- 261 nactin localisation along the MT lattice resulted in reduced ${ }_{262}$ dynein activity, we depleted p150 using siRNA mediated si- 263 lencing. As expected, we observed a reduction in the pro- 264 portion of dynein molecules that moved towards the MT mi- 265 nus ends with a concomitant increase in the proportion that 266 moved towards the plus ends (Fig. S4a-d).

The persistent association of dynactin to MTs that we 268 obseved is in contrast to in vitro observations, where recom- 269 binant human dynactin was not found to decorate pig brain- 270 derived MTs (20). Similarly recombinant budding yeast dyn- 271 actin was observed to have a weak interaction with axonemal 272 MTs (40). But when dynein was added to the mix, move- 273 ment of dynactin was observed implying that dynactin could 274 not independently bind to the MTs. However, in other in- 275 stances (41), all p150 fragments containing the CAP-Gly do- 276 main bound to the MT. Dynactin that was over expressed in 277 cells was found to bind strongly to and bundle MTs $(11,15)$. 278 In fact, dynactin-MT interactions have recently been shown 279 to be important for increasing the on-rate of dynein onto 280 MTs (42). Taken together, dynactin's MT-binding function ${ }_{281}$ is likely essential for dynein's activity in cellulo.

Endosomes remain close to MTs and move in short 284 bursts. We next sought to understand how dynein interacted ${ }_{285}$ with the third component of the active complex - the cargo 286 adaptors. The endosomal cargo adaptors, Hook proteins, have been observed to remain persistently bound to their respective cargo $(43,44)$. Therefore, we used endosomal cargo as a proxy for the cargo adaptor, which confers the additional benefit of visualising the movement of the entire cargo adaptor and cargo complex. To avoid artefacts from overexpressing fluorescently tagged Rab5 to visualise early endosomes (45), we employed cells that had taken up 10kDa dextran or EGF conjugated with Alexa 647 (Fig. S5a).

Small molecular weight dextran enters the cell through all active endocytic mechanisms via fluid phase endocytosis, and uptake is therefore relatively independent of receptormediated endocytosis $(46,47)$. On the other hand, EGF is a ligand that binds to EGF receptor on the cell membrane, which at the concentrations used in this study, is taken up by clathrin-mediated endocytosis (48). While both these cargoes eventually end up at lysosomes near the minus ends of MTs, the timescales of movement of these cargoes is vastly different, with dextran requiring hours (49) to reach lysosomes and EGF doing so in tens of minutes (50). We sought to understand how these two different kinds of cargoes could potentially employ dynein's short run times to reach the lysosomes in different time scales.

Following a short pulse and chase, we observed dextran in Rab5-positive compartments (Fig. S5b, Video S3). EGF has also been shown to be in Rab5-positive compartments within the timescales of our chase observations (51), indicating that both dextran and EGF were in early endosomes in our experiments. We probed the localisation of dextran and EGF vesicles with respect to the MT. First, we imaged 

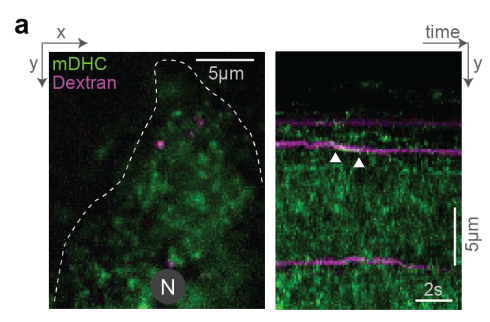

b

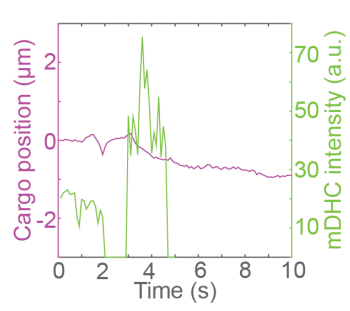

C

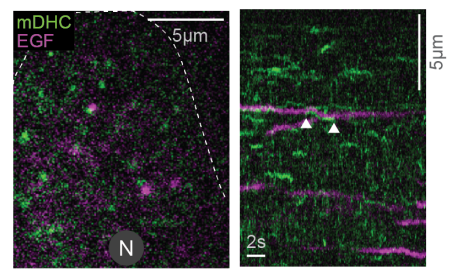

d

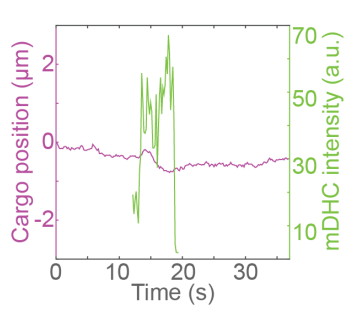

e

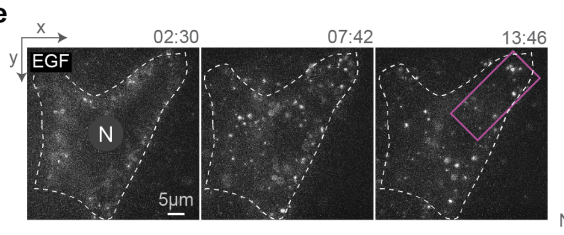
h

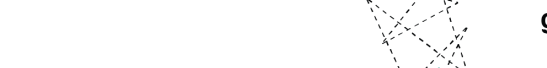

f Sampling every $5 \mathrm{~s}$ time

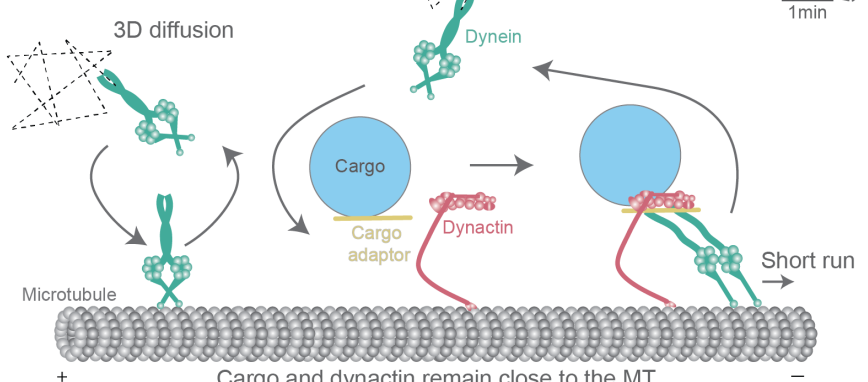

Cargo and dynactin remain close to the MT

Fig. 5. Cargo capture and dynein activation are coupled a, Image from the first frame of a time-lapse video (left) of dynein (green) and dextran (magenta), and the correspinding kymograph (right). The white arrow heads point to dynein and dextran vesicle moving together towards the minus end. $\mathbf{b}$, Plot of position vs time of the dextran vesicle (magenta) indicated in a, alongside the intensity of dynein on that vesicle (green), showing a short minus-end directed run of the vesicle upon dynein-binding. $\mathbf{c}$, Image from the first frame of a time-lapse video (left) of dynein (green) and EGF (magenta), and the corresponding kymograph (right). The white arrow heads point to dynein and EGF vesicle moving together towards the minus end. d, Plot of position vs time of the EGF vesicle (magenta) indicated in c, alongside the intensity of dynein on that vesicle (green), showing a short minus-end directed run of the vesicle upon dynein-binding. e, Images from time-lapse video of EGF entry and subsequent movement within cells. Time is indicated above the images in mm:ss. $\mathbf{f}$, Kymograph of the region indicated by the magenta rectangle in e, with the timelapse images sampled every $5 \mathrm{~s}$ showing entry of EGF and the net movement of endosomes towards the nucleus. $\mathbf{g}$, Kymograph of the trajectory marked by the white dashed rectangle in $\mathbf{f}$, where the timelapse images were sampled every $0.5 \mathrm{~s}$. In a, c, and e, 'N' marks the location/direction of the nucleus. $\mathbf{h}$, Schematic of dynein's cargo search mechanism: stochastic binding of dynein to the MT at a location proximal to the cargo-adaptor complex leads to a short minus end directed run, which terminates upon the unbinding of dynein.

dextran vesicles and MTs in live cells and observed that the ${ }_{317}$ vesicles were in proximity to the MTs (Fig. 3a). We also 318 observed that the dextran vesicles remained close to MTs ${ }_{319}$ even while they had no apparent tether to the MTs via mo- 320 tor proteins, and were therefore stationary (Video S4) in our ${ }_{321}$ minute-long live cell time-lapse images. We then used SRRF 322 to visualise Rab5 in concert with p62 in living cells, and ${ }_{323}$ observed that $\sim 70 \%$ of the Rab5 spots colocalised with p62 ${ }_{324}$ $\left(\mathrm{n}=8,795 / 12,848\right.$ vesicles from $\mathrm{N}=2$ independent experiments ${ }_{225}$ with $>20$ cells each), indicating that cargo and dynactin are 326 in proximity to each other on the MT (Fig. 3b, c). Finally, ${ }_{227}$ we employed CLEM to visualise the location of dextran and ${ }_{228}$ EGF endosomes with respect to MTs. We observed that in ${ }_{329}$ both instances, the endosomes were along MTs, indicating ${ }_{330}$ that endosomal cargo remained within $\sim 20 \mathrm{~nm}$ of MTs (dex- 331 tran: $22 \pm 16 \mathrm{~nm}$, from 3 endosomes, $\mathrm{N}=2$ cells; EGF: $10 \pm 22_{332}$ $\mathrm{nm}$, from 3 endosomes, $\mathrm{N}=2$ cells), likely along with dyn- ззз actin (Fig. 3d-i).

While the interaction of dynactin with the cargo adap- ${ }^{335}$ tor independently of dynein was discounted in earlier studies using fractionation (52), here we visualised colocalisation of dynactin with a large proportion of Rab5-labeled endosomes in close proximity to MTs (Fig. 3b).

To understand if endosomal vesicles could diffuse away ${ }_{340}$ from their original locations on the MT upon motor unbind- 341 ing, we depolymerised MTs using nocodazole and visualised ${ }_{342}$ the subsequent movement of the vesicles (Fig. S5c, Video ${ }_{343}$ S5). Confirming previous findings on Rab5-positive com- 344 partments $(30,31)$, we measured a lower diffusion coefficient 345 for the vesicles in the absence of MTs (Fig. S5d), likely im- 346 plicating the role of high intracellular crowding in constraining vesicle diffusion (31).

We next visualised dextran and EGF vesicles in 100stimelapses and observed that the directed runs were sparse (Fig. 4a, b, Videos S6, S7), with only $\sim 33 \%$ of dextran vesicles $(65 / 196)$ and $\sim 43 \%$ EGF vesicles $(92 / 214)$ moving $>1$ $\mu \mathrm{m}$ during this time (Fig. 3c). Strikingly, both the dextran and EGF vesicles displayed uninterrupted minus end-directed runs that lasted only $\sim 0.6 \mathrm{~s}$ on an average (Fig. $3 \mathrm{~d})(0.6 \pm 0.2$ $\mathrm{s}$ (mean \pm s.d.), $\mathrm{n}=196$ and 214 for dextran and EGF vesicles respectively from $\mathrm{N}=2$ independent experiments). Interestingly, the time between two consecutive runs, the pause time, reflected the time scales of minus end-directed movement of EGF and dextran, with EGF vesicles having an average pause time that was $30 \%$ shorter than that of dextran vesicles $(0.4$ s vs. $0.6 \mathrm{~s}$, Fig. $4 \mathrm{e}$ ). These results are comparable to the runand-pause behaviour observed for endosomal cargo in previous studies $(30,31)$, and will be explored further in our stochastic modelling.

\section{Dynein molecules are activated upon attachment to} dynactin and cargo on the MT. Thus far, we have discovered that dynein transiently interacts with the MTs. However, dynactin and cargo are localised together and remained close to MTs. We therefore sought to understand how dynein interacted with the dynactin-cargo complexes on MTs. First, we performed dual-colour imaging of dynein and endosomal vesicles, and observed that vesicles that had dynein signal were, as expected, more likely to move towards the minus end of MTs (Fig. S5e-g). Next, by comparing the intensity of dynein on endosomal vesicles to single molecule bind- 
a

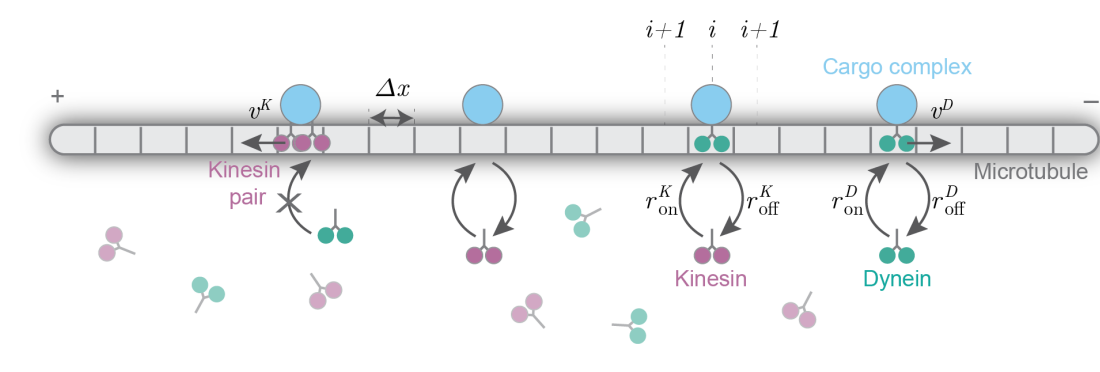

b

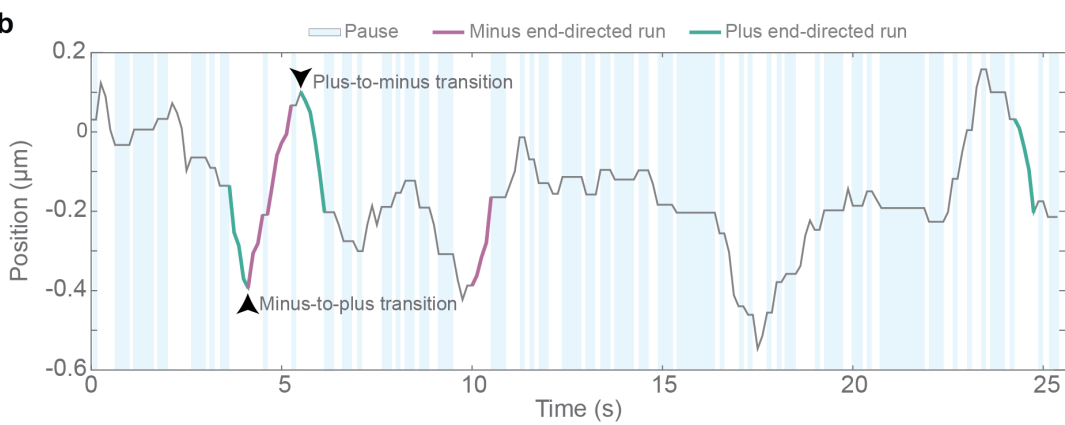

C Dextran

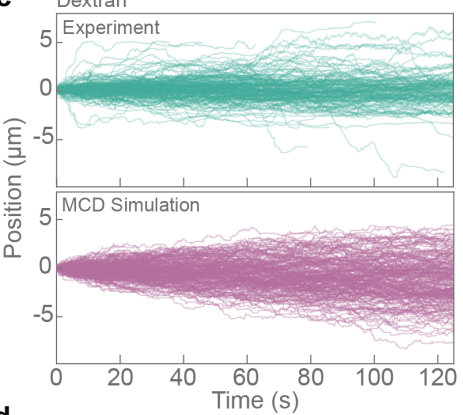

d

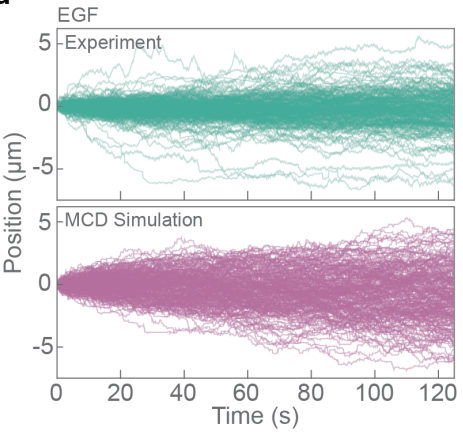

Fig. 6. MCD model for dynein's cargo capture and activation a, Schematic of the MCD model depicting the MT (grey), with lattice points separated by a distance of $\Delta x$. Cargo complexes (blue) are positioned randomly along the MT and do not diffuse away. Dynein (green) and kinesin (magenta) motors bind and unbind stochastically to the MT lattice points with rates $r_{\mathrm{on}}^{D}, r_{\mathrm{on}}^{K}$ and $r_{\mathrm{off}}^{D}, r_{\mathrm{off}}^{K}$ respectively. If a cargo-complex is present at the site of motor binding (i), the motor could hop to the adjacent site $i+1$ or $i-1$, with a rate $v^{K}$ or $v^{D}$ for kinesin and dynein respectively. Note that we if two motors are already present at a lattice point (e.g.: 'Kinesin pair'), no more motors can bind to the same site. We used periodic boundary conditions to simulate this model on an MT of length $N \Delta x$. $\mathbf{b}$, An example position vs. time trajectory of a dextran vesicle obtained from experiment (grey line), showing representative pauses (blue rectangles), minus end-directed runs (solid magenta lines), plus end-directed runs (solid green lines), and transitions from minus-to-plus and plus-to-minus end-directed runs (black arrowheads). c, Position vs. time trajectories of dextran vesicles from experimental data (green, top) and from the MCD simulation (magenta, bottom). d, Position vs. time trajectories of EGF vesicles from experimental data (green, top) and from the MCD simulation (magenta, bottom). Note that the experimental data in $\mathbf{c}$ and $\mathbf{d}$ are identical to those in Fig. $4 \mathrm{c}$ and have been reproduced here for comparison.

ing events, we estimated that, on an average, there were 1-2 374 dynein molecules bound to a vesicle (Fig. S5h). Finally, to 375 verify if single molecules of dynein could be activated and ${ }_{376}$ perform minus end-directed movement when they stochas- 377 tically bound to MTs and encountered dynactin-cargo com- 378 plexes, we performed fast dual-colour HILO imaging of sin- 379 gle dynein molecules, along with dextran and EGF vesicles. 380 In these videos (Videos S8, S9), we observed instances where ${ }_{381}$ previously stationary dextran and EGF vesicles started mov- 382 ing together with mDHC-GFP towards the MT minus ends 383 upon dynein binding (Fig. 5a-d, n=8/9 and $n=3 / 3$ events from 384 $\mathrm{N}=2$ independent experiments with at least 15 cells).

To test that the repetitive binding of dynein to an endo- ${ }^{387}$ some, followed by its short run towards the minus end was ${ }^{388}$ sufficient to effect large-scale movements towards the nu- ${ }^{389}$ cleus, we visualised the uptake and subsequent movement of 390 EGF endosomes over a $\sim 17$-min period using HILO mi- 391 croscopy. We observed robust movement of the EGF en- 392 dosomes in the net minus end direction during the duration ${ }_{393}$ of imaging (Fig. 5e, f, Videos S10). Strikingly, movements 394 that appeared as long-range at a low sampling rate (Fig. 5f), 395 showed intrinsic short runs when sampled at a higher rate 396 (Fig. 5g), similar to those seen in Fig. 4. Therefore, the 397 higher temporal resolution of our experiments enabled us to 398 discern the stop-and-go behaviour of cargo, that is ultimately ${ } 99$ sufficient to drive movement to its intracellular destination in 400 time frames consistent with those previously observed.
Conceptual model of dynein's cargo attachment and subsequent movement within cells. Taken together, we observed that: (i) single molecules of dynein bind and unbind stochastically with MTs (Fig. 1b), (ii) if a dynactin-cargo complex is found close to this attachment location of dynein to the MT, a minus end-directed run of the dynactin-cargomotor tripartite complex is effected (Fig. 5a-d), (iii) the detachment of dynein from the cargo concludes this run (Fig. $5 \mathrm{~h}$ ), (iv) the detached dynein motor is free to diffuse back into the cytoplasm, whereas dynactin and the cargo remain paused and remain close to the MT (Fig. 3), (v) the longrange movement of endosomal cargo requires the repeated binding-unbinding of dynein to the dynactin-cargo complex, (vi) the resulting motion of the cargo consists of short minus (and plus) end-directed runs punctuated by long pauses (Fig. $4 c)$.

Several remarks are in order. First, the steps outlined above constitute an effective intermittent mechanism to ensure cargo transport by stochastic motor dynamics. Such strategies have been described in the past for transcription factors seeking specific sequences along the DNA $(53,54)$. Second, since cytoplasmic dynein is the only minus enddirected motor that participates in membrane trafficking in many cell types, how dynein interacts with and transports different types of cargo is an interesting question. Recent research suggests that cargo specific adaptors like BicD2 (for Rab6-positive cargo), and Hook1/3 (for Rab5-positive cargo) have differential interaction with dynein (20). These cargo 
a

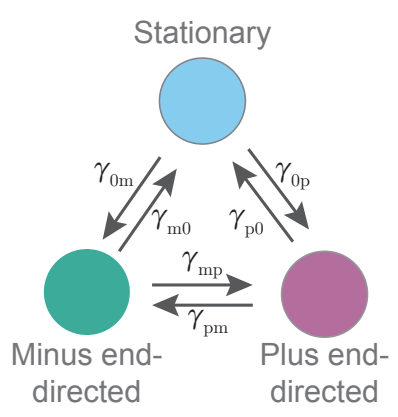

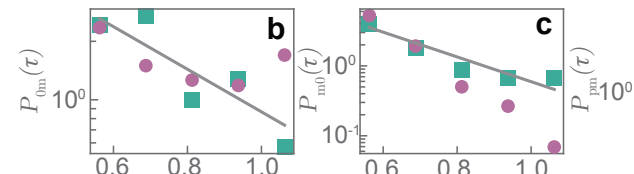

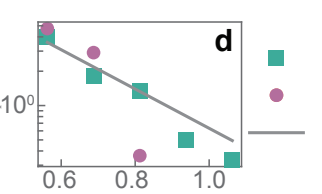

Experiment MCD Simulation RTP model
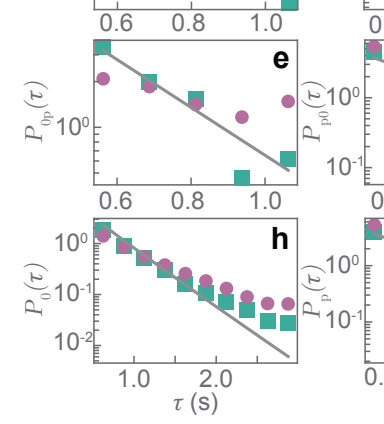

Fig. 7. The 3-state RTP model and its fits for the movement of dextran vesicles. a, Schematic of the RTP model depicting the 3 states: stationary, minus end-directed and plus end-directed, and the transition rates between them, i.e. $\gamma_{0 \mathrm{~m}}, \gamma_{\mathrm{m} 0}-$ rates of transition from the stationary to minus end-directed state and vice versa; $\gamma_{0 \mathrm{p}}$, $\gamma_{\mathrm{p} 0}-$ rate of transition from the stationary to the plus end-directed state and vice versa; $\gamma_{\mathrm{mp}}, \gamma_{\mathrm{pm}}$-rates of transition from the minus end-directed to the plus end-directed state and vice versa. In b-g, experimental data (green squares) are compared with the output from MCD simulations (magenta circles) and the predictions of the RTP model (grey lines) for the probability distributions of the cargo waiting time $(\tau)$ while transitioning from the stationary to the minus end-directed state (b), minus end-directed to stationary (c), plus to minus end-directed (d), stationary to plus end-directed (e), plus end-directed to stationary (f), and minus end-directed to plus end-directed (g). Further, the probability distributions of the pause-times $(\mathbf{h})$, the run times in the plus end-directed state (i), and the run times in the minus end-directed state (j) are shown in $\mathbf{h}-\mathbf{j}$, while $\mathbf{k}$ and $\mathbf{I}$ show the distributions of the the run lengths $(l)$ in the plus end-directed and minus end-directed states respectively. The solid grey lines represent fits to the experimental data from the RTP model. The MCD model results allowed pairs of motors to transport cargo-complexes, i.e., $M=2$. Note that he plots (b-I) are in log-linear scale.

adaptors could modulate the interaction time of dynein with ${ }_{437}$ the cargo thereby leading to different kinetics. Such stochas- 438 tic binding and unbinding allow the same dynein molecule ${ }_{439}$ to sample and interact with a wide range of cargo, akin to 440 round robin scheduling. Third, differences in the internalisation route for cargo bound by the same adaptor, such as $\mathrm{EGF}_{441}$ and dextran as seen in this study, could also alter the num- ${ }_{442}$ ber of cargo adaptors on the cargo, which in turn changes the ${ }_{43}$ motor on-rates to the cargo. Movement to the plus or mi- ${ }_{444}$ nus ends of MTs could therefore also be tuned by slightly ${ }_{445}$ increasing the bias in one direction, for example by increas- ${ }_{446}$ ing the number of adaptors for dynein on a cargo destined ${ }_{447}$ towards the minus end. Fourth, cellular morphology is likely ${ }_{448}$ to play a significant role in the kinetics of motor attachment ${ }_{449}$ and detachment from the MT. For instance, in highly polar, ${ }_{450}$ narrow axons of neuronal cells, reattachment of dynein to ${ }_{45}$ cargo localised close to the MT is likely to occur frequently ${ }_{452}$ due to the confinement, and therefore reduced space in which ${ }_{453}$ dynein could diffuse. This would result in higher on rates for ${ }_{454}$ the motor to the cargo, and therefore more processive cargo ${ }_{455}$ trajectories. Finally, it is very likely that a similar mechanism ${ }_{450}$ is at work in transporting plus end directed cargo by kinesin ${ }_{457}$ motors. More generally, both dyneins and kinesins can si- ${ }_{458}$ multaneously affect cargo movement, even for those cargo ${ }_{459}$ that are transported towards the minus end on the average ${ }_{460}$ such as dextran and EGF.

Our experimental techniques - HILO, SD and SRRF, and 462 CLEM - provided high spatiotemporal data for the dynam- 463 ics of motors and dynactin-cargo complexes. In fact, this not 464 only allowed us to quantify the stochastic kinetics of individ- 465 ual dynein motors to extract its detachment rates (Fig. 1f), but 466 also allowed us to explore the long-time dynamics of cargo ${ }_{467}$ with high precision (Fig. 4c). As such, we sought to quan- 468 tify the statistical dynamics of endosomal cargo by measuring ${ }_{469}$ the distributions of the cargo residence time of both dextran 470 and EGF in the moving and paused states. Before discussing these results in detail (Fig. 7-9), we develop stochastic models at two levels of descriptions that can quantitatively account for these distributions of cargo dynamics.

\section{A stochastic model captures the kinetics of motor-} driven cargo movements. To study the stochastic dynamics of motors and cargo units capable of activating the motor (which include dynactin and adaptor for dynein, henceforth referred to as 'cargo complexes'), we modelled the MT as a discrete one-dimensional lattice of $N$ binding-sites each of size $\Delta x(=0.05 \mu \mathrm{m})$ (Fig. 6a). Our model included both dyneins and kinesins interacting with the MT and cargo complexes. At each site $i$ of the MT, exchange kinetics with a large cytoplasmic reservoir caused dynein (kinesin) motors to bind at a Poisson rate $r_{\mathrm{on}}^{D}\left(r_{\mathrm{on}}^{K}\right)$ to the MT and unbind from the MT at a Poisson rate $r_{\text {off }}^{D}\left(r_{\text {off }}^{K}\right)$ to the cytoplasm. Note that this binding-unbinding process was stochastic and occurred uniformly throughout the length of the MT. If a cargo complex was found at the binding location of a motor protein, then the motor-cargo complex translocated along the MT with a speed $v^{D}$ towards the minus end in the case of dyneins and with a speed $v^{K}$ towards the plus end in the case of kinesins. Dynein (kinesin) motors detached from the MT after a mean residence time $\sim 1 / r_{\text {off }}^{D}\left(\sim 1 / r_{\text {off }}^{K}\right)$, which also ended the motion of the cargo complex. From this point on, the cargo complex remained stationary, but still in proximity to the MT. In other words, we completely neglected the 3D diffusion of the cargo-adaptor complexes since our data suggested that cargo diffusion is negligible (Fig. S5d). Furthermore, we only considered a single cargo complex at a time and disregarded any steric interactions between them. The chemical master equation describing this Markovian stochastic process is described in detail in the Supplementary Text. When we restricted the number of motors that could be bound 
to each site of the MT $(M)$ to one, this stochastic motor- 528 cargo complex dynamics model (referred to as the 'MCD ${ }_{529}$ model' henceforth) generated cargo trajectories that under- 530 went pauses, minus end-directed and plus end-directed runs 531 (Fig. S6). However, in this instance, the only transitions that 532 occurred were between paused and moving (plus or minus) ${ }_{53}$ states of the cargo complex, which were not truly reflective 534 of our experimental observations.

Typical experimental trajectories of the cargo showed ${ }_{536}$ discrete paused and moving states (Fig. 6b for dextran). 537 In addition to the transitions between pauses and runs, we 538 observed direct transitions between minus end-directed runs ${ }_{539}$ and plus end-directed runs. This, however, is not possible 540 unless the cargo complex was moved by at least a pair of ${ }_{541}$ motors consisting of one dynein and one kinesin. In other 542 words, our experimental data (Fig. 6b) indicated that there ${ }_{543}$ were antagonistic pairs of motor proteins bound to the cargo 544 complex (see Fig. S6 for the output of the MCD model with ${ }_{545}$ $M=1$ ). In fact, our experiments with dual imaging data of 546 single molecules of dynein and cargo indicated that there ${ }_{547}$ were one to two dyneins bound to the cargo (Fig. S5h). It 548 was thus reasonable to assume that we would also have pairs 549 of kinesins, and dynein-kinesin pairs bound to a cargo com- 550 plex. Note that cargo translocation could be effected by both single motors as well as pairs of motors.

We therefore extended our MCD model to the case where a maximum of $M=2$ motors could be bound to each site of the MT. Note that even with $M=2$, we assumed that the motors hopped at the same speeds, i.e., at rates $v^{D} / \Delta x$ and $v^{K} / \Delta x$, across the lattice sites of the MT. In particular, we also assumed that the antagonistic pair could move in either direction at the appropriate rates. Further, we neglected any cooperative binding between multiple motors. We discuss in detail the chemical master equation associated with this model in the Supplementary Text, and also its stochastic simulation via the Gillespie algorithm $(55,56)$. With values for the parameters $r_{\mathrm{on}}^{D}, r_{\mathrm{on}}^{K}, r_{\mathrm{off}}^{D}, r_{\mathrm{off}}^{K}, v^{D}$ and $v^{K}$ that compared reasonably well with their estimates from experiments (see table in Fig. 9c), the MCD model generated cargo trajectories that were comparable to empirical results (Fig. 6c and d). However, taking advantage of our long-term and high temporal resolution imaging data, we next asked whether the model could quantitatively account for the residence time distributions of the cargo in the various states, and if there was a simplified description for the dynamics of the cargo complexes that themselves behaved as "super-motors", displaying motion which was markedly different from simple Brownian motion on the MT.

\section{The emergent dynamics of cargo is captured by a 3-s-} tate run-and-tumble model. Typical trajectories, such as the one shown in Fig. 6b, revealed short periods of directed 551 motion of the cargo complexes interspersed with frequent 552 pause events. This kind of stochastic motion was not char- 553 acteristic of the simple, and well-known, thermal Brownian 554 motion. Rather, this movement was reminiscent of the clas- 555 sic run-and-tumble motion wherein bacteria run in almost 556 straight line paths for a certain run-duration after which they 557 tumble while being stationary for a tumble-duration, and then run again in a randomly chosen direction (57). Statistical physics models of such run-and-tumble particles (RTP) have revealed very interesting features of these persistent and active random walks (58-60). However, in these well-studied RTP models, the tumble is, typically considered to be an instantaneous event. Several studies have explored models, with three distinct states- two moving states (with movements in opposite directions), and a paused state- for cargo that arise from underlying motor movement $(61,62)$.

Our MCD model discussed in the previous section led to an emergent picture for the dynamics of the cargo complexes as RTPs moving on a one-dimensional MT with three distinct states: minus end-directed, paused and plus end-directed. In more detail, these states resulted from the following situations: (i) a minus-end directed run of the cargo complex occurred when there were motor configurations dominated by dyneins, (ii) cargo complexes were paused when there were no motors (of either kind) within a proximal region on the MT, and (iii) a plus-end directed run occurred when kinesins were the primary movers of the cargo complexes. As such, the effective movement of the cargo was captured by three discrete states with distinct transition rates between them.

We captured the dynamics of the cargo complexes discussed above in a 3-state RTP model. Specifically, as depicted in Figure 7a, stationary cargo could transition to the plus (minus) end-directed state at a rate $\gamma_{0 \mathrm{p}}\left(\gamma_{0 \mathrm{~m}}\right)$, while moving cargo could transition to the stationary state at rates $\gamma_{\mathrm{m} 0}$ (from the minus end-directed state) and $\gamma_{\mathrm{p} 0}$ (from the plus end-directed state). Further, we considered direct transitions between the plus and minus end-directed states at rates $\gamma_{\mathrm{pm}}$ and $\gamma_{\mathrm{mp}}$ respectively, since these transitions were apparent in our experimental data (Fig. 6b). In the plus enddirected state, the cargo translocated with a velocity $u=+u^{\mathrm{p}}$ and in the minus end-directed state, the translocation velocity was $u=-u^{\mathrm{m}}$. In the stationary state, by definition, the cargo was immobile, i.e., $u=0$. Therefore, denoting $\left(\mathcal{P}_{\mathrm{m}}, \mathcal{P}_{0}, \mathcal{P}_{\mathrm{p}}\right)$ as the probabilities of finding the cargo in the minus end-directed, stationary and plus end-directed states respectively, the master equations governing their Markovian time-evolution were:

$$
\frac{d}{d t}\left(\begin{array}{c}
\mathcal{P}_{\mathrm{m}} \\
\mathcal{P}_{0} \\
\mathcal{P}_{\mathrm{p}}
\end{array}\right)=\Gamma\left(\begin{array}{l}
\mathcal{P}_{\mathrm{m}} \\
\mathcal{P}_{0} \\
\mathcal{P}_{\mathrm{p}}
\end{array}\right)
$$

where the transition matrix $\Gamma$ was:

$$
\Gamma=\left(\begin{array}{ccc}
-\gamma_{\mathrm{m} 0}-\gamma_{\mathrm{mp}} & \gamma_{0 \mathrm{~m}} & \gamma_{\mathrm{pm}} \\
\gamma_{\mathrm{m} 0} & -\gamma_{0 \mathrm{~m}}-\gamma_{0 \mathrm{p}} & \gamma_{\mathrm{p} 0} \\
\gamma_{\mathrm{mp}} & \gamma_{0 \mathrm{p}} & -\gamma_{\mathrm{p} 0}-\gamma_{\mathrm{pm}}
\end{array}\right)
$$

Note that in these emergent discrete states, the cargo complexes moved with the appropriate velocity. In other words, the position $x$ of the cargo complex changed with time according to $d x / d t=u$, where the velocity $u$ could take either of the three possibilities $\left\{-u^{\mathrm{m}}, 0, u^{\mathrm{p}}\right\}$.

The stochastic dynamics captured by equation Eq. (1) predicts that the distribution $P_{\mathrm{ab}}(\tau)$ of the residence time 

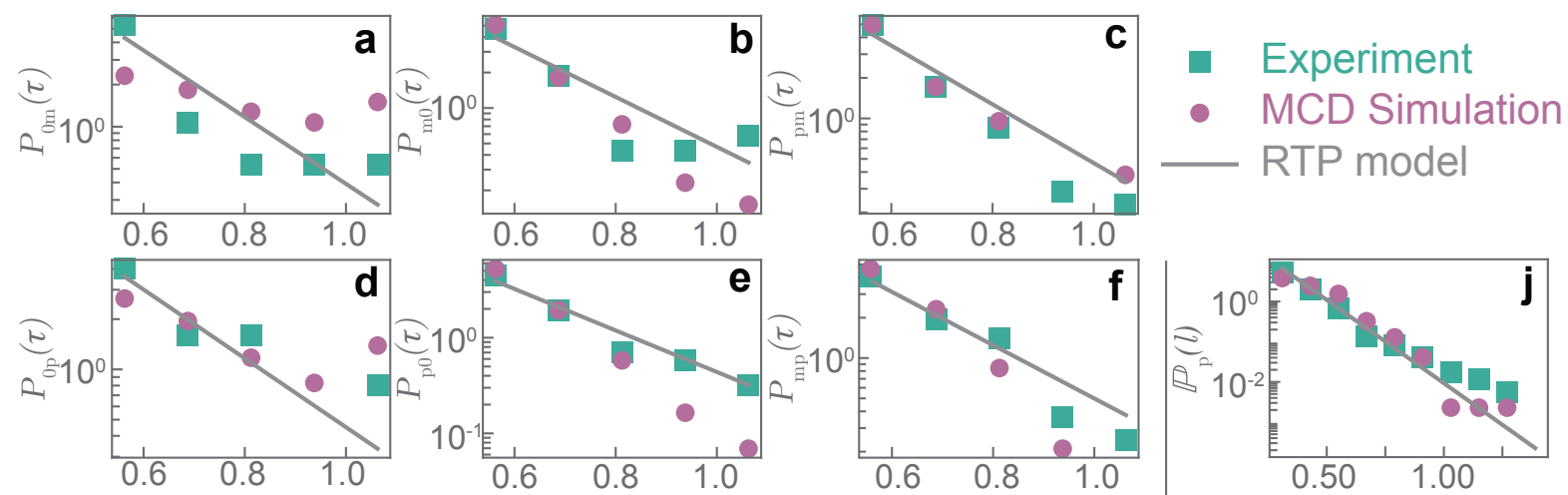
- RTP model
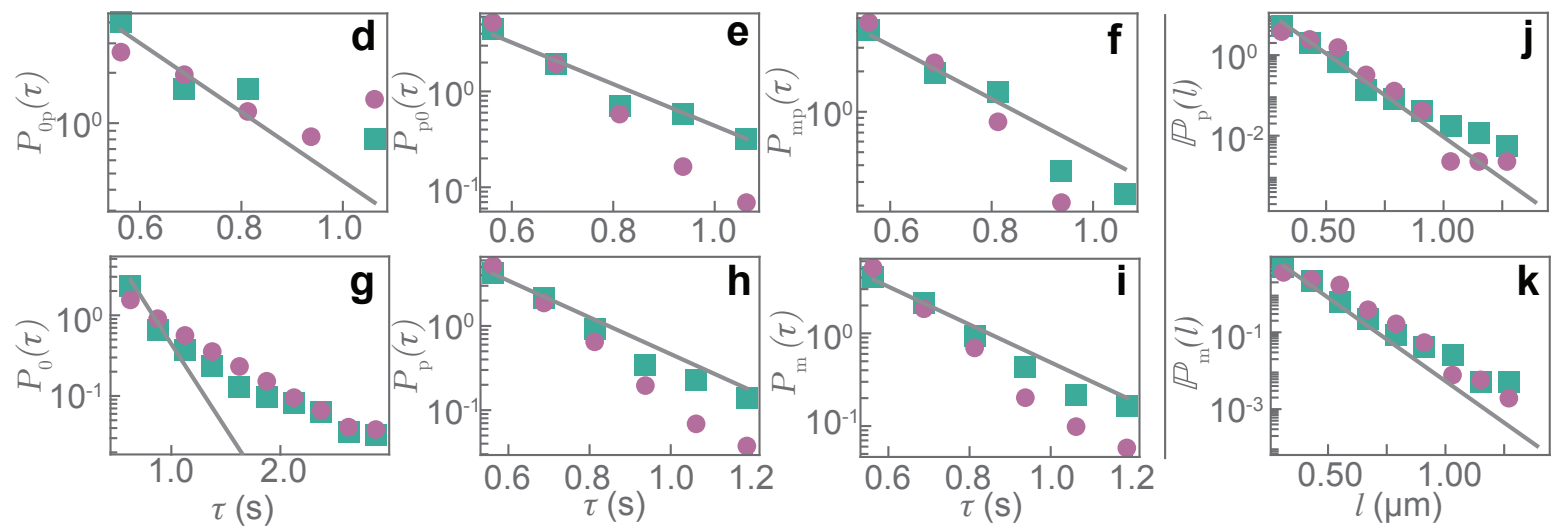

Fig. 8. Fits of the 3-state RTP model for EGF vesicle trajectories. In a-f, experimental data (green squares) are compared with the output from MCD simulations (magenta circles) and the predictions of the RTP model (grey lines) for the probability distributions of the cargo waiting time $(\tau)$ while transitioning from the stationary to the minus end-directed state (a), minus end-directed to stationary (b), plus to minus end-directed (c), stationary to plus end-directed (d), plus end-directed to stationary (e), and minus end-directed to plus end-directed (f). Further, the probability distributions of the pause-times (g), the run times in the plus end-directed state (h), and the run times in the minus end-directed state (i) are shown in $\mathbf{g}-\mathbf{i}$, while $\mathbf{j}$ and $\mathbf{k}$ show the distributions of the the run lengths $(l)$ in the plus end-directed and minus end-directed states respectively. The solid grey lines represent fits to the experimental data from the RTP model. The MCD model results allowed pairs of motors to transport cargo-complexes, i.e., M=2. Note that the plots a-k are in log-linear scale.

(equivalently waiting time) in the a-state before jumping to 589 the b-state is an exponential distribution with the rate con- 590 stant $\gamma_{\mathrm{ab}}$ (here $\mathrm{a}, \mathrm{b}$ could take either of the values $\mathrm{m}, 0$ or 591 p). For instance, the distribution of the residence times of the 592 cargo complex in the paused state before transitioning to the ${ }_{593}$ minus end-directed state would be $P_{0 \mathrm{~m}}(\tau)=A_{0 \mathrm{~m}} e^{-\gamma_{0 \mathrm{~m}} \tau}$, 594 where $A_{0 \mathrm{~m}}$ is an amplitude factor. Similar considerations 595 applied to the waiting time distribution for the other transi- 596 tions.

We next asked whether our high-temporal resolution, ${ }_{590}$ long-term data for the positions of the cargo complexes was ${ }_{600}$ consistent with the waiting time distributions obtained from ${ }_{601}^{600}$ this coarse-grained 3-state RTP model. To this end, we partitioned the trajectory of the cargo complexes into discrete ${ }_{603}^{602}$ states (Fig. 6b, see Methods), measured the waiting time for each transition depicted in Fig. 7a, and fitted exponential functions to the probability density functions of the resi- 605 dence times $\tau$. We found a good match between our empiri- 606 cal data and the distributions $P_{\mathrm{ab}}(\tau)$ as shown in Fig. 7(b-g) ${ }_{607}$ for the case of dextran vesicles. We employed this proce- 608 dure to extract the effective rate constants $\gamma_{\mathrm{ab}}$ for a transi- 609 tion of the cargo complex from the a-state to the b-state 610 (see the table in Fig. 9c). Notice that the cargo could exit ${ }_{611}$ the stationary/paused state via two independent pathways: ${ }_{612}$ transitioning into the minus end-directed state or transition- 613 ing into the plus end-directed state. As such, the distribu- ${ }_{614}$ tion of the pause times was a mixed distribution $P_{0}(\tau)={ }_{615}$ $w_{0} P_{0 \mathrm{~m}}(\tau)+\left(1-w_{0}\right) P_{0 \mathrm{p}}(\tau)$, where $0 \leq w_{0} \leq 1$ is a weight ${ }_{616}$ factor. To check whether this prediction worked, we fixed ${ }_{617}$ the rates $\gamma_{0 \mathrm{p}}$ and $\gamma_{0 \mathrm{~m}}$ to the values obtained by the fits in ${ }_{618}$ Fig. 7b and Fig. 7e respectively, and fit the form of $P_{0}(\tau){ }_{619}$ to the empirical data. The excellent fit seen in Fig. 7h confirms the validity of our 3-state RTP model. Similar considerations applied for the distribution $P_{\mathrm{m}}(\tau)=w_{\mathrm{m}} P_{\mathrm{m} 0}(\tau)+$ $\left(1-w_{\mathrm{m}}\right) P_{\mathrm{mp}}(\tau)$ of minus end-directed run times (Fig. 7i), and the distribution $P_{\mathrm{p}}(\tau)=w_{\mathrm{p}} P_{\mathrm{p} 0}(\tau)+\left(1-w_{\mathrm{p}}\right) P_{\mathrm{pm}}(\tau)$ of plus end-directed run times (Fig. $7 \mathrm{j}$ ). Further, in the minus (plus) end-directed states, we measured the distribution of the run lengths $\mathbb{P}_{\mathrm{m}}(l)$ and $\left(\mathbb{P}_{\mathrm{m}}(l)\right)$. Since the cargo complexes only moved when they were in the $m-$ or $p$-states, the run length distributions were expected to be related to the run time distributions. Specifically, we expected: $\mathbb{P}_{\mathrm{m}}(l)=\left[\omega_{\mathrm{m}} P_{\mathrm{m} 0}\left(l / u^{\mathrm{m}}\right)+\left(1-\omega_{\mathrm{m}}\right) P_{\mathrm{mp}}\left(l / u^{\mathrm{m}}\right)\right] / u^{\mathrm{m}}$, and $\mathbb{P}_{\mathrm{p}}(l)=\left[\omega_{\mathrm{p}} P_{\mathrm{p} 0}\left(l / u^{\mathrm{p}}\right)+\left(1-\omega_{\mathrm{p}}\right) P_{\mathrm{pm}}\left(l / u^{\mathrm{p}}\right)\right] / u^{\mathrm{p}}$. The excellent fits of the empirical data to these distributions seen in (Fig. 7j-k) allowed us to extract the emergent speeds $u^{\mathrm{m}}$ and $u^{\mathrm{p}}$ of the cargo complexes.

We obtained more compelling evidence to this emergent picture when we were able to match the results of the MCD simulation, depicted in Fig. 6a, for the various residence time and run length distributions of the empirical data and the RTP model. We repeated this procedure for the case of EGF vesicles and obtained similar agreement between the empirical data, the MCD simulation and the RTP model as shown in Fig. 8. The parameter values both in our MCD simulations and those obtained in the RTP model are available in Fig. 9c. The predictive power of our approach was revealed when we examined the dependence of the average run length $\langle l\rangle$ as a function of the run time $\tau$ in the case of both dextran and EGF. Since all parameters in the RTP model were known, we compared the empirical data and the MCD simulation data with the predictions $\langle l\rangle=u^{\mathrm{p}} \tau$ and $\langle l\rangle=-u^{\mathrm{m}} \tau$ for plus end- 
a

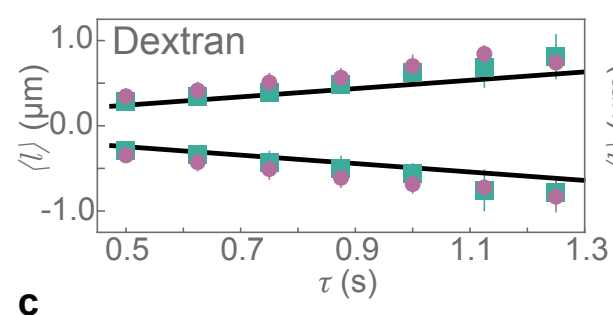

b

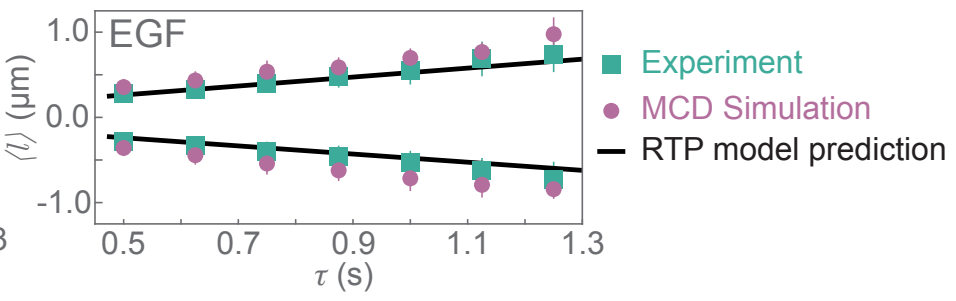

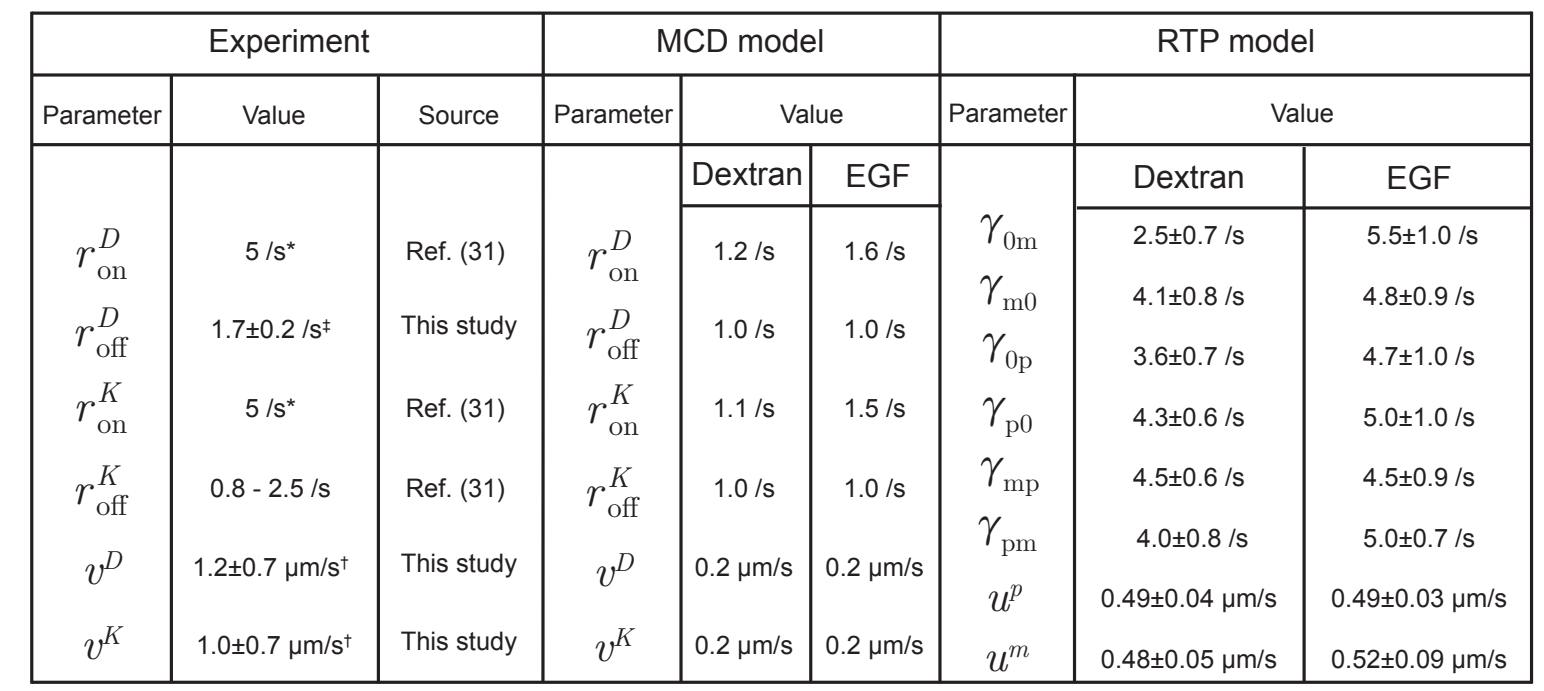

* Estimated from comparing experimental data to a stochastic model, $\$ 95 \%$ confidence interval, ${ }^{+}$Standard deviation

Fig. 9. Run lengths of cargo scale with run time, Plot of average run length $\langle l\rangle$ as a function of run time $\tau$ for dextran vesicles (a) and EGF vesicles (b), with data from experiments (green squares) and output from the MCD model with $M=2$ (magenta circles). The black solid line represents the prediction of the RTP model using the parameter listed in the table below. c Table containing the parameters that were measured for single dynein and kinesin molecules in this study and other experiments ('Experiments'), those that were employed in the MCD model ('Stochastic model'), and those for the cargo movement that obtained from fits to the experimental data from the RTP model ('RTP model') for both dextran and EGF.

directed and minus end-directed runs respectively. The re- 645 markable agreement seen in Fig. 9a and b confirmed that 646 our simulation and modelling approaches provide an accu- 647 rate description of the experimental data. So too, the scaling ${ }_{648}$ of emergent cargo velocities with run time points to the par- 649 ticipation of at least 2 motors in the cargo trafficking process. 650 Indeed, the output from the MCD model with $M=1$ did not match the experimental data (Fig. S6) or fit the RTP model ${ }_{651}$ (Figs. S7, S8a).

It is thus apparent that our MCD model, which explic- 653 itly included the dynamics of motors, led to a coarse-grained 654 emergent view wherein the dynamics of the cargo complexes 655 were governed by a 3-state RTP model. However, an im- 656 portant point to note is that it is a non-trivial task to find 657 analytical relations between the emergent parameters at the 658 RTP level $\left(\gamma_{\mathrm{p} 0}, \gamma_{\mathrm{m} 0}, \gamma_{0 \mathrm{~m}}, \gamma_{0 \mathrm{p}}, \gamma_{\mathrm{pm}}, \gamma_{\mathrm{mp}}, u^{\mathrm{m}}\right.$ and $\left.u^{\mathrm{p}}\right)$ and ${ }_{659}$ the parameters in the model $\left(r_{\mathrm{on}}^{D}, r_{\mathrm{off}}^{D}, r_{\mathrm{on}}^{K}, r_{\mathrm{off}}^{K}, v^{D}\right.$ and $\left.v^{K}\right) .660$ Nevertheless, the parameters used in our simulations revealed 661 that the on-rates for both dynein and kinesin are higher by 662 $\sim 30 \%$ for EGF as compared to dextran. Remarkably, our 663 experimental data showed that EGF vesicles had an average 664 pause time which was $\sim 30 \%$ shorter than that of dextran 665 vesicles (Fig. 4e). This difference in on-rates and thereby 666 the pause times, could have arisen from an increased number ${ }_{667}$ of adaptors for dynein (and kinesin) on EGF-containing vesi- 668 cles compared to dextan-containing vesicles, and will be the subject of future studies. Together, we developed a framework for considering cargo movement in light of a 3-state RTP model which could be used to infer not only underlying motor kinetics, but also predict long-term cargo movement and fates, such as in differential cargo sorting.

\section{Conclusion}

In this work, we established a technique to visualise single molecules of dynein in living cells, and observed their interaction with the MT, and other components of the tripartite complex required for its activation. We discovered distinct localisation and kinetics of dynein, dynactin and the cargo complex: While dynein interacted stochastically and transiently with the MT, dynactin and cargo remained persistently bound and in proximity to the MT. When dynein interacted with a dynactin-cargo complex upon MT-binding, the entire complex moved towards the minus end of MTs in a short run lasting a little over half a second. Repeated rounds of such stochastic interactions of the motor with the MT and cargo complex are required for long-range movement of cargoes.

We measured single-molecule parameters pertaining to dynein, and used these and other parameters available in literature to develop a MCD model for cargo trajectories based on assumptions from experiments. The experimental data 
and output from the MCD model for two different kinds of 725 cargo - dextran and EGF vesicles - were in good agreement. 726 Importantly, the experimental data and MCD model output 727 quantitatively agree with the predictions of a 3-state RTP ${ }_{728}$ model. Taken together, we demonstrate that stochastic in- 729 teractions of motors with MTs and cargo complexes are suf- 730 ficient to elicit complex cargo trafficking behaviour in living ${ }_{731}$ cells.

\section{Methods}

Cell culture. HeLa cells were cultured in Dulbecco's ${ }^{735}$ Modified Eagle Media (DMEM) containing 100 units $/ \mathrm{mL}^{736}$ Penicillin, $0.1 \mathrm{mg} / \mathrm{mL}$ Streptomycin, $2 \mathrm{mM}$ L-Glutamine, $400 \mu \mathrm{g} / \mathrm{mL}$ Geneticin (HiMedia Laboratories) and sup- ${ }^{739}$ plemented with $10 \%$ fetal bovine serum (Sigma Aldrich). Cells were grown in an incubator at $37^{\circ} \mathrm{C}$ under $5 \% \mathrm{CO}_{2}$. During live-cell imaging, cells were maintained in livecell imaging solution $\left(140 \mathrm{mM} \mathrm{NaCl}, 2.5 \mathrm{mM} \mathrm{KCl}, 1.8^{742}\right.$ $\mathrm{mM} \mathrm{CaCl} 2,1 \mathrm{mM} \mathrm{MgCl} 2,4 \mathrm{mg} / \mathrm{mL}$ D-Glucose, $20 \mathrm{mM}_{744}^{743}$ HEPES) at $37^{\circ} \mathrm{C}$ using a stage-top incubator (Oko Labs).

RNAi experiments. For all RNAi experiments, cells were ${ }^{746}$ first grown in glass-bottom imaging dishes (Cat. no. 81518, Ibidi) for $48 \mathrm{~h}$, transfected with the appropriate concentration of siRNA using Jet Prime transfection reagent (Cat. no. ${ }^{749}$ 114, Polyplus) and imaged after $48 \mathrm{~h}$. For hDHC RNAi the following siRNA sequence was used: 5'-ACA-UCA-ACA- ${ }^{751}$ UAG-ACA-UUC-A-3'10. For p150 RNAi the following siRNA sequences were used: 5'-GUA-CUU-CAC-UUGUGA-UGA-A-3', 5'-GAU-CGA-GAG-ACA-GUU-AUU- ${ }^{754}$ A-3' (14). siRNA oligonucleotides were procured from ${ }^{755}$ Eurogentec, Belgium. To quantify the knockdown of pro- ${ }^{756}$ teins, the cells were lysed 48 hours after siRNA transfection ${ }^{757}$ to obtain protein solution and SDS-PAGE, western blotting ${ }^{758}$ was performed. The following primary antibodies were ${ }^{759}$ used to visualise proteins of interest and controls: Rabbit ${ }^{760}$ DYNC1H1 Polyclonal Antibody (PA5-49451, Invitrogen, ${ }^{761}$ $0.5 \mu \mathrm{g} / \mathrm{mL}$ ), Rabbit Dynactin 1 Polyclonal Antibody (PA5- ${ }^{762}$ 37360, Invitrogen, $0.5 \mu \mathrm{g} / \mathrm{mL}$ ), Mouse GAPDH Loading ${ }^{763}$ Control Antibody (MA5-15738, Invitrogen, $0.5 \mu \mathrm{g} / \mathrm{mL}$ ), ${ }^{764}$ Rabbit Actin Polyclonal Antibody (ab8227, AbCam, ${ }^{765}$ $0.5 \mu \mathrm{g} / \mathrm{mL}$ ). The following secondary antibodies were ${ }^{766}$ used: Anti-Rabbit HRP (31460, Invitrogen, $0.16 \mu \mathrm{g} / \mathrm{mL})^{767}$ and Anti-Mouse HRP (62-6520, Invitrogen, $0.3 \mu \mathrm{g} / \mathrm{mL}$ ).

Immunofluorescence. To immunostain p150 and tubulin, ${ }^{770}$ cells were first grown for $48 \mathrm{~h}$ in glass-bottom imaging dishes ${ }^{771}$ (Cat. no. 81518, Ibidi) and fixed in ice-cold methanol at ${ }^{772}$ $-20^{\circ} \mathrm{C}$ for $3 \mathrm{~min}$. Cells were washed thrice in phosphate ${ }^{773}$ buffered saline (PBS) for 5 min each and incubated in block- 774 ing buffer (5\% BSA in PBS) for $60 \mathrm{~min}$. Subsequently, the ${ }^{775}$ cells were incubated with p150 and $\alpha$-tubulin antibodies in 776 antibody dilution buffer (1\% BSA in PBS) for 60 min. Cells ${ }^{777}$ were then washed thrice in PBS for 5 mins and incubated 778 with secondary antibodies in antibody dilution buffer for 45779 min. At the end of incubation with secondary antibodies, the 780 cells were washed with PBS and imaged immediately. The 781 following primary antibodies were used. Rabbit Dynactin 1 Polyclonal Antibody (PA5-21289, Invitrogen, $2 \mu \mathrm{g} / \mathrm{mL}$ ), Mouse $\alpha$ Tubulin Monoclonal Antibody (32-2500, Invitrogen, $2 \mu \mathrm{g} / \mathrm{mL}$ ). The secondary antibodies used were Donkey Anti-Rabbit A555 Antibody (A31572, Invitrogen, 0.4 $\mu \mathrm{g} / \mathrm{mL}$ ) and Goat Anti-Mouse A647 Antibody (A28181, Invitrogen. $2 \mu \mathrm{g} / \mathrm{mL}$ ).

To immunostain p150 and p62, p62 and $\alpha$-Tubulin and EB1 and $\alpha$-Tubulin the following method was used. Cells were first grown for $48 \mathrm{~h}$ in glass bottomed imaging dishes and fixed in ice-cold methanol at $-20^{\circ} \mathrm{C}$ for $3 \mathrm{~min}$. Subsequently, the cells were washed thrice in PBS for $5 \mathrm{~min}$ each and incubated in antibody dilution buffer (2\% BSA, 0.1\% Triton-X100, in PBS) for $10 \mathrm{~min}$. Next, the cells were incubated in antibody dilution buffer containing the appropriate antibodies for $60 \mathrm{~min}$. Cells were washed thrice in PBS for $5 \mathrm{~min}$ each and incubated with appropriate secondary antibodies in antibody dilution buffer for $30 \mathrm{~min}$. At the end of incubation with secondary antibodies, the cells were washed well in PBS and imaged immediately. The following primary antibodies were used. Rabbit Dynactin 1 Polyclonal Antibody (PA5-21289, Invitrogen, $2.5 \mu \mathrm{g} / \mathrm{mL}$ ), Rabbit Alpha Tubulin Monoclonal Antibody (PA5-22060, Invitrogen, $2 \mu \mathrm{g} / \mathrm{mL}$ ), Mouse Dynactin 4 Monoclonal Antibody (MA5-17065, Invitrogen, $2 \mu \mathrm{g} / \mathrm{mL}$ ) and Mouse EB1 Monoclonal Antibody (412100, Invitrogen, $2 \mu \mathrm{g} / \mathrm{mL}$ ). The secondary antibodies used were Donkey Anti-Rabbit A647 Antibody (A32795, Invitrogen, $2 \mu \mathrm{g} / \mathrm{mL}$ ) and Goat Anti-Mouse A555 Antibody (A21422, Invitrogen. $2 \mu \mathrm{g} / \mathrm{mL}$ ).

To immunostain Rab5 and p62, cells were first fixed in 4\% Paraformaldehyde (PFA) for 15 mins. The cells were washed well in PBS and incubated for $45 \mathrm{~min}$ in antibody staining solution $(0.2 \%$ Saponin, $0.1 \%$ BSA and $0.02 \%$ Sodium Azide in PBS) containing primary antibodies against Rab5 and p62. Subsequently, cells were washed well in PBS and incubated for $45 \mathrm{~min}$ in antibody staining solution containing appropriate secondary antibodies. Cells were washed well in PBS and imaged immediately. The following primary antibodies were used. Rabbit Rab5 Monoclonal Antibody (3547, Cell Signaling Technology, $14 \mu \mathrm{g} / \mathrm{mL}$ ), Mouse Dynactin 4 Monoclonal Antibody (MA5-17065, Invitrogen, $2 \mu \mathrm{g} / \mathrm{mL}$ ). The secondary antibodies used were Donkey Anti-Rabbit A647 Antibody (A32795, Invitrogen, $2 \mu \mathrm{g} / \mathrm{mL}$ ) and Goat AntiMouse A555 Antibody (A21422, Invitrogen. $2 \mu \mathrm{g} / \mathrm{mL}$ ).

Dextran and EGF uptake. To visualise fluorescent dextran vesicles, HeLa cells that were grown in glass-bottom imaging dishes (Cat. no. 81518, Ibidi) for $48 \mathrm{~h}$ were transferred to serum-free DMEM for $4 \mathrm{~h}$ in a $37^{\circ} \mathrm{C} \mathrm{CO}_{2}$ incubator. Subsequently, the cells were pulsed for $10 \mathrm{~min}$ in complete DMEM containing $200 \mu \mathrm{g} / \mathrm{mL}$ A647-Dextran (D-22914, Invitrogen). Cells were then washed well with live-cell imaging solution before proceeding for microscopy. In all experiments, imaging was completed within 45 min of dextran uptake.

To visualise fluorescent EGF vesicles, HeLa cells that were grown in glass-bottom imaging dishes (Cat. 
no. P35G-1.5-14-C, Mattek) for $24 \mathrm{~h}$ were transferred ${ }_{839}$ to serum-free DMEM for $1 \mathrm{~h}$ in a $37^{\circ} \mathrm{C} \mathrm{CO}_{2}$ incubator. 840 Cells were washed in serum-free DMEM, then incubated 841 with 1 nM EGF (Cat. No. E9644, Sigma-Aldrich) la- 842 belled with Alexa647 (Cat. No. ab269823, Abcam) ${ }_{843}$ in phenol-free, serum-free DMEM for a minimum of ${ }_{844}$ 8 minutes prior to imaging. In all experiments, cells ${ }_{845}$ were imaged between 8-20 minutes of EGF uptake. ${ }_{846}$

Transfection. To visualise fluorescently tagged proteins, 849 cells were transfected with the appropriate plasmids using ${ }_{850}$ Jet Prime Transfection Reagent (Cat. no. 114, Polyplus). ${ }_{851}$ $3 \mathrm{~h}$ after transfection, the cells were washed well in PBS, ${ }_{852}$ grown in complete DMEM at $37^{\circ} \mathrm{C}$ and imaged $\sim 20 \mathrm{~h}_{853}$ later. The following plasmids were used in this study. (i) ${ }_{854}$ mCherry-tubulin was a gift from Mariola Chacon, TUD, ${ }_{855}$ Dresden. (ii) Gal4T-mCherry was a gift from Thomas ${ }_{856}$ Pucadyil, IISER Pune. (iii) mCherry-DCTN1 was a gift from ${ }_{857}$ Prof. Kozo Tanaka, Tohoku university, Japan. (iv) mCherry- ${ }_{858}$ Rab5 was a gift from Gia Voeltz (Addgene plasmid - 49201).

Microscopy. Imaging was performed on a Nikon Ti2E ${ }_{861}$ inverted microscope equipped with a Toptica MLE laser 862 combiner, Nikon H-TIRF Module, Yokogawa CSU-X1 863 spinning disk module, an Andor iXon 897 EMCCD 864 camera and an Oko Lab stage top incubator. The mi- 865 croscope was controlled using Nikon NIS Elements 866 software or Micromanager (63). Alternately, a Zeiss 867 ELYRA controlled using Zen Black software was used. 868

HILO microscopy and particle tracking. For HILO microscopy, a Nikon 100X 1.49NA TIRF objective was used. 871 We optimised the HILO microscopy setting for each cell: 872 First, to avoid overexpression artefacts, we visualised cells 873 expressing low levels of mDHC-GFP. The diameter of the il- 874 luminated area was kept constant at $30 \mu \mathrm{m}$ and the long axis 875 of cell was aligned perpendicular to the excitation laser. Fi- 876 nally, we adjusted the angle of incidence of the excitation 877 laser, such that the fluorescent spots in a plane $\sim 0.5 \mu \mathrm{m}$ from 878 the cover slip appeared bright and distinct. The incidence an- 879 gle and orientation of excitation laser beam were adjusted us- 880 ing the H-TIRF module and the laser power (50 $\mathrm{mW}$ at fiber) ${ }_{881}$ was kept at $40 \%$. To visualise single molecules of dynein, ${ }_{882}$ time-lapse images were acquired at $50 \mathrm{fps}$ with $20 \mathrm{~ms}$ expo- ${ }_{883}$ sure per frame.

To improve the signal-to-noise ratio, a five-frame slid- 885 ing average of the time-lapse images was used for particle 886 tracking. In these videos, the appearance and subsequent dis- 887 appearance of intensity at a particular location was classified 888 as a binding event. Such binding events were visually identi- 889 fied and tracked from the start to end frame using Low Light 890 Tracking tool (64) in Fiji/ImageJ (65). The fluorescent spots 891 were classified as stationary, minus-end moving or plus-end 892 moving by marking a region close to the nucleus as the mi- 893 nus end and calculating the displacement of fluorescent spots 894 with respect to the minus end. Particles moving away from 895 the nucleus for $>0.32 \mu \mathrm{m}$ were classified as plus-end moving, and particles with displacement $>0.32 \mu \mathrm{m}$ towards the nucleus were minus-end directed, and particles with net displacement $<0.32 \mu \mathrm{m}$ were classified as stationary.

For dual channel imaging of dynein and cargo, videos were acquired at $20 \mathrm{fps}$ with $25 \mathrm{~ms}$ exposure per channel for dynein and dextran (in cells depleted of $\mathrm{hDHC}$ using RNAi), and at $6 \mathrm{fps}$ with $33 \mathrm{~ms}$ exposure per channel for dynein and EGF. To observe dynein-dextran interaction with a better signal-to-noise ratio, a two-frame sliding average of timelapse images was used.

For $100 \mathrm{~s}$ dextran and EGF imaging experiments, an alpha Plan-Apochromat 100x/1.46 Oil Elyra TIRF objective was used. Consistent HILO setting were used across all longterm dextran and EGF uptake experiments. Fluorophores were excited using a $642 \mathrm{~nm}$ laser $(150 \mathrm{~mW}$ at source) at a TIRF angle of $63^{\circ}$. Exposure times of $50 \mathrm{~ms}$ with a frame interval of $100 \mathrm{~ms}$ were used (total frame time, $125 \mathrm{~ms}$ with frame transfer), and imaging undertaken for 1000 frames (125 s in total). The movement of dextran vesicles was classified in the following way: the movement between two consecutive frames was classified as zero if it was less than $20 \mathrm{~nm}$ (the tracking accuracy of low light tracking tool under the imaging conditions used (64).). Then, displacement for four consecutive frames towards or away from the nucleus was classified as a minus or plus end-directed run, and a zero displacement for 2 or more consecutive frames was classified as a pause. The same criteria for runs and pauses was used for analysing the trajectories obtained from the MCD model.

Spinning disk (SD) confocal microscopy and image analysis. To quantify the correlation between expression level of dynein and its clustering, z-stack images of live HeLa cells expressing mDHC-GFP were acquired using a 60x, 1.4 NA objective with $50 \mathrm{~ms}$ exposure. The intensity comparison was done for the lowest plane at which clusters were distinctly visible. The dynamics of mCherry-p150 in cells were visualised by acquiring 60-s long movies of the cell with a 1.4 NA 60x objective, $100 \mathrm{~ms}$ exposure per frame and $1 \mathrm{~s}$ interval between frames. The dynamics of dextran vesicles along the MTs was visualised by acquiring 30-s long movies of the cell with a 60x, 1.4 NA objective, $100 \mathrm{~ms}$ sequential exposure/channel and $1 \mathrm{~s}$ interval between frames.

The SD+SRRF images were obtained by taking the mean of the radiality map of 100 images of a single field acquired under a 100x, 1.49NA objective with the SD confocal microscopy setup. The radiality magnification in SRRF was set to 4 for all experiments. To visualise the association between MTs and dextran vesicles in live cells, the SRRF acquisition settings used were $20 \mathrm{~ms}$ exposure, ring radius of 0.5 for MTs and 3 for dextran. To visualise p62 with tubulin and EB1 with tubulin, $20 \mathrm{~ms}$ exposure and ring radius of 0.5 was used. To visualise p150 with tubulin, and p150 with p62 the SRRF acquisition settings used were $100 \mathrm{~ms}$ exposure, ring radius of 1. Finally, to visualise p62 with Rab5, 20ms exposure was used in both channels. A ring radius of 0.5 and 3 were used for p62 and Rab5, respectively. 

available under aCC-BY-NC-ND 4.0 International license.

The cooccurrence of p150 with p62 was quantified 953 by first thresholding SRRF images of $\mathrm{p} 150$ for the top 954 $5 \%$ of intensity. The number of p150 spots was counted 955 $N_{p 150}$, and a mask was created. Then, the SRRF image 956 of p62 in the same cells was combined with the p150 957 mask using AND operation in Fiji. The resultant image 958 was thresholded and the number of spots counted $\left(N_{p 62}\right) .959$ $N_{p 62} / N_{p 150} * 100$ gave the percentage of p150 spots with 960 p62. A similar procedure was used to quantify the per-961 centage of p62 spots that occurred on the MT and also the 962 percentage of Rab5 spots that were associated with p62. 963 To quantify the effect of p150 knock-down on the lev-964 els of p150, p62 along the MT, ROIs were drawn 965 along distinct MT segments that were randomly cho-966 sen in SRRF images of MT, and the mean intensi-967 ties of p150 or p62 in these ROIs were measured.

Correlative Light and Electron Microscopy. HeLa cells were grown for 24 hours on gridded glass-bottom imaging dishes (Cat. No. P35G-1.5-14-CGRD, Mattek), following which 973 cells were transfected with EGFP-tubulin (Cat. No. 56450, ${ }_{974}$ AddGene) overnight. Cells were transferred into serum-free, ${ }_{975}$ phenol free DMEM for $1 \mathrm{~h}$, following which A647-dextran ${ }_{976}$ or EGF-647 were added to cells for $10 \mathrm{~min}$. Cells were extensively washed in PBS and fixed in 4\% PFA containing 978 $0.2 \%$ glutaraldehyde (Cat. No. 354400, Sigma-Aldrich) ${ }_{979}$ for $1 \mathrm{~h}$ at room temperature. Following fixation, cells ${ }_{980}$ were dyed with MitoTracker Orange CMTMRos (Cat. No. ${ }_{981}$ M7510) to fluorescently label mitochondria through the cell ${ }_{982}$ volume, then imaged on a Zeiss LSM 800 with Airyscan ${ }_{983}$ detector, with a pixel size of $42.5 \mathrm{~nm}$ and z-slice spacing of $150 \mathrm{~nm}$. The MitoTracker-stained mitochondria served ${ }^{984}$ as markers for correlation and registration of the relevant ${ }_{986}^{985}$ confocal z-slice with that of the corresponding TEM z-slice ${ }^{987}$ based on identification of mitochondrial morphology and ${ }_{989}^{988}$ distribution at each slice (Fig. S8). After imaging, cells 990 were further fixed in $2.5 \%$ glutaraldehyde in $0.1 \mathrm{M}$ sodium ${ }_{992}^{991}$ cacodylate for $1 \mathrm{~h}$ at room temperature. All the subsequent ${ }^{993}$ processing steps were carried out in a BioWave microwave ${ }_{995}^{994}$ (Pelco). Post fixation, samples were washed with cacodylate 996 buffer, additionally fixed in reduced osmium (1\% osmium ${ }^{997}$ tetroxide- $1.5 \%$ potassium ferricyanide) solution followed by buffer wash, resuspended in $2 \%$ aqueous $\mathrm{OsO}_{4}$ (osmium 998 tetroxide) (ProScitech). Washed samples were then stained with $2 \%$ w/v Phosphotungstic acid (PTA) in 30\% ethanoliooo at $60^{\circ}$ for $1 \mathrm{~h}$, followed with a $30 \%$ ethanol wash at room ${ }_{100}^{100}$ temperature. Samples were then stained with $2 \%$ aqueous 1003 uranyl acetate prepared in $30 \%$ ethanol, incubated at $4{ }^{\circ} \mathrm{C}_{1005}^{1004}$ for $1 \mathrm{~h}$. Serial dehydration was then continued at this point 1006 in increasing percentages of ethanol, following which cells ${ }_{0008}^{1007}$ were serially infiltrated with Durcupan ACM (Cat. No.1009 44610, Sigma). Fresh $100 \%$ resin was then added and $d_{1011}^{1010}$ polymerised at $60^{\circ} \mathrm{C}$ overnight. Ultrathin sections were 1012 cut on an ultramicrotome (UC6: Leica) and imaged at at $_{1014}^{1013}$ $100 \mathrm{kV}$ on a JEOL1400 transmission electron microscope 1015 fitted with a Phurona EMSIS CMOS camera using a $5 \times 5^{1016}$ stitching matrix. Light and electron microscopy images were ${ }_{1018}$ subsequently overlayed and correlated using the Correlia plugin for Fiji/ImageJ (66) and is briefly described here. Using the signal from the orange fluorescent mitochondrial dye, a TEM section of the cell 65 -nm thick was correlated with the best fit confocal z-slice within the realms of the confocal slice thickness of $150 \mathrm{~nm}$. An overlay of the TEM image and corresponding confocal image showing the mitochondrial signal only at the relevant z-slice was created for cells that had taken up both A647-dextran and A647-EGF. Once the overlay confirmed the right cell volume, then the green signal from EGFP-tubulin and the red signal from A647-dextran or EGF-647 were overlayed onto the same TEM image and this enabled visualisation of the proximity of dextran and EGF endosomes with respect to MTs.

Curve fitting and data visualisation. For the intensity fits in Fig. 1c, the intensity values of tracked dynein molecules was plotted as a histogram. This distribution was fit by a sum of two Gaussians with the same standard deviation $(\sigma)$. The smaller mean was constrained between 15-30 and the larger mean was constrained between 35-50. For the exponential fit in Fig. 1f, the probability vs residence time $(\tau)$ histogram of dynein on the MTs was obtained from the lengths of all single molecule binding events. From this $P(\tau)=1$-cumulative frequency of the residence time was obtained, and was fit to $\lambda e^{-\lambda t}$ to obtain $\lambda$, the off-rate from MTs. The average residence time was given by $1 / \lambda$. The fits in Figs. 7, 8 and 9 were similarly obtained using in-built Python functions. All data was plotted using MATLAB (MathWorks) or Python. Figures panels were prepared using Adobe Illustrator.

\section{ACKNOWLEDGEMENTS}

We thank LA Chacko for constructive comments on the manuscript. VA is supported by EMBL Australia, and was supported by extramural funding from the Wellcome Trust/Department of Biotechnology-India Alliance (grant IA/18/1/503607), and the Women Excellence Award from the Science and Engineering Research Board, India, intramural funding from the Indian Institute of Science, and the RI Mazumdar Young Investigator Award and Department of Science and Technology (India) INSPIRE Faculty Award. KVK acknowledges support from the Department of Atomic Energy, Government of India under project no. RTI4001, the Department of Biotechnology, India, through a Ramalingaswami re-entry fellowship and the Max Planck Society through a Max-Planck-Partner-Group at ICTS-TIFR. This research was supported in part by the International Centre for Theoretical Sciences (ICTS-TIFR) for participating in the online program "Thirsting for Theoretical Biology" (code: ICTS/ttb2021/1).

\section{References}

1. Höök $P$ and Vallee RB. The dynein family at a glance. Journal of cell science, 119(Pt 21): 4369-4371, nov 2006. ISSN 0021-9533. doi: 10.1242/JCS.03176.

2. Victoria J. Allan. Cytoplasmic dynein. Biochemical Society Transactions, 39(5):1169-1178, oct 2011. ISSN 0300-5127. doi: 10.1042/BST0391169.

3. Kristen J Verhey and Jennetta W Hammond. Traffic control: regulation of kinesin motors. Nature Reviews Molecular Cell Biology, 10(11):765-77, nov 2009. ISSN 1471-0080. doi: $10.1038 / \mathrm{nrm} 2782$.

4. S J King and T A Schroer. Dynactin increases the processivity of the cytoplasmic dynein motor. Nature cell biology, 2(1):20-4, jan 2000. ISSN 1465-7392. doi: 10.1038/71338.

5. Richard B Vallee, Richard J Mckenney, and Kassandra M Ori-mckenney. Multiple modes of cytoplasmic dynein regulation. Nature Publishing Group, 14(3):224-230, 2012. ISSN 1465-7392. doi: 10.1038/ncb2420.

6. Michael a. Cianfrocco, Morgan E. DeSantis, Andres E. Leschziner, and Samara L. ReckPeterson. Mechanism and Regulation of Cytoplasmic Dynein. Annual Review of Cell and Developmental Biology, 31(1):annurev-cellbio-100814-125438, 2015. ISSN 1081-0706. doi: 10.1146/annurev-cellbio-100814-125438.

7. Vaishnavi Ananthanarayanan. Activation of the motor protein upon attachment: Anchors weigh in on cytoplasmic dynein regulation. BioEssays, 38(6):514-525, 2016. ISSN 1521 1878. doi: 10.1002/bies.201600002.

8. John T. Canty and Ahmet Yildiz. Activation and Regulation of Cytoplasmic Dynein. Trends 
bioRxiv preprint doi: https://doi.org/10.1101/2021.04.05.438428; this version posted November 1, 2021. The copyright holder for this preprint (which was not certified by peer review) is the author/funder, who has granted bioRxiv a license to display the preprint in perpetuity. It is made available under aCC-BY-NC-ND 4.0 International license.

in Biochemical Sciences, 45(5):440-453, may 2020. ISSN 0968-0004. doi: 10.1016/J.TIBS.1105 2020.02.002.

9. Trina A Schroer. DYNACTIN. Annual Review of Cell and Developmental Biology, 20(1):1107 759-779, nov 2004. ISSN 1081-0706. doi: 10.1146/annurev.cellbio.20.012103.094623. 1108

10. Steven R Gill, Trina A Schroer, Illya Szilak, Eric R Steuer, Michael P Sheetz, and Don W1109 Cleveland. Dynactin, a conserved, ubiquitously expressed component of an activator ofi110 vesicle motility mediated by cytoplasmic dynein. Journal of Cell Biology, 115(6):1639-1650,1111 dec 1991. ISSN 0021-9525. doi: 10.1083/jcb.115.6.1639.

11. N. J. Quintyne, S. R. Gill, D. M. Eckley, C. L. Crego, D. A. Compton, and T. A. Schroer.1113 Dynactin is required for microtubule anchoring at centrosomes. Journal of Cell Biology, 1471114 (2):321-334, 1999. ISSN 00219525. doi: 10.1083/jcb.147.2.321

12. Caterina Valetti, Dawn M Wetzel, Michael Schrader, M Josh Hasbani, Steven R Gill,1116 Thomas E Kreis, and Trina A Schroer. Role of dynactin in endocytic traffic: Effects of 1117 dynamitin overexpression and colocalization with CLIP-170. Molecular Biology of the Cell,1118 10(12):4107-4120, dec 1999. ISSN 10591524. doi: 10.1091/mbc.10.12.4107.

13. K T Vaughan, S H Tynan, N E Faulkner, C J Echeverri, and R B Vallee. Colocalization of 1120 cytoplasmic dynein with dynactin and CLIP-170 at microtubule distal ends. Journal of celh 121 science, 112 ( Pt 1:1437-47, 1999. ISSN 0021-9533.

14. Peter Watson and David J. Stephens. Microtubule plus-end loading of p150Glued is me-1123 diated by EB1 and CLIP-170 but is not required for intracellular membrane traffic in mam-1124 malian cells. Journal of Cell Science, 119(13):2758-2767, 2006. ISSN 00219533. doi:1125 $10.1242 /$ jcs.02999.

15. Patricia S. Vaughan, Pedro Miura, Matthew Henderson, Belinda Byrne, and Kevin T1127 Vaughan. A role for regulated binding of p150Glued to microtubule plus ends in or-1128 ganelle transport. Journal of Cell Biology, 158(2):305-319, 2002. ISSN 15408140. doi:1129 $10.1083 /$ jcb.200201029.

16. Daniël Splinter, David S. Razafsky, Max A. Schlager, Andrea Serra-Marques, Ilya Grig-1131 oriev, Jeroen Demmers, Nanda Keijzer, Kai Jiang, Ina Poser, Anthony A. Hyman, Casper C.1132 Hoogenraad, Stephen J. King, and Anna Akhmanova. BICD2, dynactin, and LIS1 cooperate1133 in regulating dynein recruitment to cellular structures. Molecular Biology of the Cell, 23(21):1134 4226-4241, nov 2012. ISSN 1059-1524. doi: 10.1091/mbc.e12-03-0210.

17. Casper $C$ Hoogenraad and Anna Akhmanova. Bicaudal D Family of Motor Adaptors : Link-1136 ing Dynein Motility to Cargo Binding. Trends in Cell Biology, 26(5):327-340, 2016. ISSN1137 0962-8924. doi: 10.1016/j.tcb.2016.01.001.

18. Samara L. Reck-Peterson, William B. Redwine, Ronald D. Vale, and Andrew P Carter. The1139 cytoplasmic dynein transport machinery and its many cargoes. Nature Reviews Moleculan 140 Cell Biology, 19(6):382-398, jun 2018. ISSN 1471-0072. doi: 10.1038/s41580-018-0004-3.1141

19. Mara A Olenick and Erika L. F. Holzbaur. Dynein activators and adaptors at a glance.1142 Journal of Cell Science, 132(6):1-7, mar 2019. ISSN 1477-9137. doi: 10.1242/jcs.227132. 1143

20. R. J. McKenney, W. Huynh, M. E. Tanenbaum, G. Bhabha, and R. D. Vale. Activation1144 of cytoplasmic dynein motility by dynactin-cargo adapter complexes. Science, 345(6194):1145 337-341, jun 2014. ISSN 0036-8075. doi: 10.1126/science.1254198.

21. Max A Schlager, Ha Thi Hoang, Linas Urnavicius, Simon L Bullock, and Andrew P Carter. In1 147 vitro reconstitution of a highly processive recombinant human dynein complex. The EMBO1148 Journal, 33(17):1855-1868, sep 2014. ISSN 0261-4189. doi: 10.15252/embj.201488792. 1149

22. Takayuki Torisawa, Muneyoshi Ichikawa, Akane Furuta, Kei Saito, Kazuhiro Oiwa, Hiroaki1 150 Kojima, Yoko Y Toyoshima, and Ken'ya Furuta. Autoinhibition and cooperative activation1151 mechanisms of cytoplasmic dynein. Nature cell biology, 16:1118-1124, sep 2014. ISSN1152 1476-4679. doi: 10.1038/ncb3048.

23. Kai Zhang, Helen E. Foster, Arnaud Rondelet, Samuel E. Lacey, Nadia Bahi-Buisson,1154 Alexander W. Bird, and Andrew P. Carter. Cryo-EM Reveals How Human Cytoplasmic1 155 Dynein Is Auto-inhibited and Activated. Cell, 169(7):1303-1314.e18, 2017. ISSN 10974172.1156 doi: 10.1016/j.cell.2017.05.025.

24. Saikat Chowdhury, Stephanie A Ketcham, Trina A Schroer, and Gabriel C Lander. Structural1 158 organization of the dynein-dynactin complex bound to microtubules. Nature Structural and 159 Molecular Biology, 22(4):345-347, apr 2015. ISSN 1545-9993. doi: 10.1038/nsmb.2996. 1160

25. Vladislav Belyy, Max A. Schlager, Helen Foster, Armando E. Reimer, Andrew P. Carter, and1161 Ahmet Yildiz. The mammalian dynein-dynactin complex is a strong opponent to kinesin in a1 162 tug-of-war competition. Nature Cell Biology, 18(9):1018-1024, 2016. ISSN 14764679. doi:1163 $10.1038 / \mathrm{ncb} 3393$.

26. Ina Poser, Mihail Sarov, James R.A. Hutchins, Jean Karim Hériché, Yusuke Toyoda, Andrei1 165 Pozniakovsky, Daniela Weigl, Anja Nitzsche, Björn Hegemann, Alexander W. Bird, Laurence1 166 Pelletier, Ralf Kittler, Sujun Hua, Ronald Naumann, Martina Augsburg, Martina M. Sykora,1167 Helmut Hofemeister, Youming Zhang, Kim Nasmyth, Kevin P. White, Steffen Dietzel, Karl1168 Mechtler, Richard Durbin, A. Francis Stewart, Jan Michael Peters, Frank Buchholz, and1169 Anthony A. Hyman. BAC TransgeneOmics: A high-throughput method for exploration of1170 protein function in mammals. Nature Methods, 2008. ISSN 15487091. doi: 10.1038/nmeth.1171 1199.

27. Makio Tokunaga, Naoko Imamoto, and Kumiko Sakata-Sogawa. Highly inclined thin illumi-1173 nation enables clear single-molecule imaging in cells. Nature methods, 5(2):159-61, feb1174 2008. ISSN 1548-7105. doi: 10.1038/nmeth1171.

28. Vaishnavi Ananthanarayanan, Martin Schattat, Sven K Vogel, Alexander Krull, Nenad Pavin,1176 and Iva M Tolić-Nørrelykke. Dynein Motion Switches from Diffusive to Directed upon Cortical1 177 Anchoring. Cell, 153(7):1526-1536, jun 2013. ISSN 00928674. doi: 10.1016/j.cell.2013.05.1178 020 .

29. V Ananthanarayanan and Iva Marija Tolić. Single-molecule imaging of cytoplasmic dynein1180 in vivo. Methods in Cell Biology, 125:1-12, 2015. doi: 10.1016/bs.mcb.2014.10.001. 1181

30. Neftali Flores-Rodriguez, Salman S. Rogers, David A. Kenwright, Thomas A. Waigh,1182 Philip G. Woodman, and Victoria J. Allan. Roles of Dynein and Dynactin in Early Endo-1183 some Dynamics Revealed Using Automated Tracking and Global Analysis. PLOS ONE, 61184 (9):e24479, sep 2011. ISSN 1932-6203. doi: 10.1371/journal.pone.0024479.

31. Allison L. Zajac, Yale E. Goldman, Erika L F Holzbaur, and E. Michael Ostap. Local cy-1186 toskeletal and organelle interactions impact molecular-motor-driven early endosomal traf-1187 ficking. Current Biology, 23(13):1173-1180, 2013. ISSN 09609822. doi: 10.1016/j.cub.1188 2013.05.015.

32. Ambarish Kunwar, Suvranta K. Tripathy, Jing Xu, Michelle K. Mattson, Preetha Anand,1190
Roby Sigua, Michael Vershinin, Richard J. McKenney, Clare C. Yu, Alexander Mogilner, and Steven P. Gross. Mechanical stochastic tug-of-war models cannot explain bidirectiona lipid-droplet transport. Proceedings of the National Academy of Sciences, 108(47):1896018965, nov 2011. ISSN 0027-8424. doi: 10.1073/PNAS.1107841108.

33. Jennifer L. Ross, Karen Wallace, Henry Shuman, Yale E. Goldman, and Erika L.F. Holzbaur. Processive bidirectional motion of dynein-dynactin complexes in vitro. Nature Cell Biology, 8(6):562-570, may 2006. ISSN 1465-7392. doi: 10.1038/ncb1421.

34. Linas Urnavicius, Kai Zhang, Aristides G Diamant, Carina Motz, Max A Schlager, Minmin Yu, Nisha A Patel, Carol V Robinson, and Andrew P Carter. The structure of the dynactin complex and its interaction with dynein. Science (New York, N.Y.), 347(6229):1441-6, mar 2015. ISSN 1095-9203. doi: 10.1126/science.aaa4080.

35. Armen J. Moughamian, Gregory E. Osborn, Jacob E. Lazarus, Sandra Maday, and Erika L.F. Holzbaur. Ordered recruitment of Dynactin to the microtubule plus-end is required for efficient initiation of retrograde axonal transport. Journal of Neuroscience, 33 (32):13190-13203, 2013. ISSN 02706474. doi: 10.1523/JNEUROSCI.0935-13.2013.

36. Hwajin Kim, Shuo-Chien Ling, Gregory C Rogers, Comert Kural, Paul R Selvin, Stephen L Rogers, and Vladimir I Gelfand. Microtubule binding by dynactin is required for microtubule organization but not cargo transport. The Journal of cell biology, 176(5):641-51, feb 2007. ISSN 0021-9525. doi: 10.1083/jcb.200608128.

37. Nireekshit Addanki Tirumala and Vaishnavi Ananthanarayanan. Role of Dynactin in the Intracellular Localization and Activation of Cytoplasmic Dynein. Biochemistry, 59(2):156162, 2020. ISSN 15204995. doi: 10.1021/acs.biochem.9b00772.

38. Nils Gustafsson, Siân Culley, George Ashdown, Dylan M. Owen, Pedro Matos Pereira, and Ricardo Henriques. Fast live-cell conventional fluorophore nanoscopy with ImageJ through super-resolution radial fluctuations. Nature Communications, 7, 2016. ISSN 20411723. doi: 10.1038/ncomms 12471 .

39. Takuya Kobayashi and Takashi Murayama. Cell Cycle-Dependent Microtubule-Based Dynamic Transport of Cytoplasmic Dynein in Mammalian Cells. PLoS One, 4(11), 2009. doi: 10.1371/journal.pone.0007827.

40. J. R. Kardon, S. L. Reck-Peterson, and R. D. Vale. Regulation of the processivity and intracellular localization of Saccharomyces cerevisiae dynein by dynactin. Proceedings of the National Academy of Sciences, 106(14):5669-5674, 2009. ISSN 0027-8424. doi: 10. 1073/pnas.0900976106.

41. Tara L. Culver-Hanlon, Stephanie A. Lex, Andrew D. Stephens, Nicholas J. Quintyne, and Stephen J. King. A microtubule-binding domain in dynactin increases dynein processivity by skating along microtubules. Nature Cell Biology, 8(3):264-270, 2006. ISSN 14657392. doi: $10.1038 / n c b 1370$.

42. Paulomi Sanghavi, Pankaj Kumar, Ankit Roy, M. S. Madhusudhan, and Roop Mallik. On and off controls within dynein-dynactin on native cargoes. Proceedings of the National Academy of Sciences, 118(23):e2103383118, jun 2021. ISSN 0027-8424. doi: 10.1073/ pnas. 2103383118 .

43. Ewa Bielska, Martin Schuster, Yvonne Roger, Adokiye Berepiki, Darren M. Soanes, Nicholas J. Talbot, and Gero Steinberg. Hook is an adapter that coordinates kinesin-3 and dynein cargo attachment on early endosomes. Journal of Cell Biology, 204(6):989-1007, mar 2014. ISSN 1540-8140. doi: 10.1083/jcb.201309022.

44. Theodoros Matanis, Anna Akhmanova, Phebe Wulf, Elaine Del Nery, Thomas Weide, Tatiana Stepanova, Niels Galjart, Frank Grosveld, Bruno Goud, Chris I. De Zeeuw, Angelika Barnekow, and Casper C. Hoogenraad. Bicaudal-D regulates COPI-independent Golgi-ER transport by recruiting the dynein-dynactin motor complex. Nature Cell Biology, 4(12):986992, 2002. ISSN 14657392. doi: 10.1038/ncb891.

45. Erik Nielsen, Fedor Severin, Jonathan M. Backer, Anthony A. Hyman, and Marino Zerial. Rab5 regulates motility of early endosomes on microtubules. Nature Cell Biology, 1(6): 376-382, oct 1999. ISSN 1465-7392. doi: 10.1038/14075.

46. Li L, Wan T, Wan M, Liu B, Cheng R, and Zhang R. The effect of the size of fluorescent dextran on its endocytic pathway. Cell biology international, 39(5):531-539, may 2015. ISSN 1095-8355. doi: 10.1002/CBIN.10424.

47. Joshua J. Rennick, Angus P. R. Johnston, and Robert G. Parton. Key principles and methods for studying the endocytosis of biological and nanoparticle therapeutics. $\mathrm{Na}$ ture Nanotechnology 2021 16:3, 16(3):266-276, mar 2021. ISSN 1748-3395. doi: 10.1038/s41565-021-00858-8.

48. Lai Kuan Goh, Fangtian Huang, Woong Kim, Steven Gygi, and Alexander Sorkin. Multiple mechanisms collectively regulate clathrin-mediated endocytosis of the epidermal growth factor receptor. Journal of Cell Biology, 189(5):871-883, may 2010. ISSN 1540-8140. doi: 10.1083/jcb.201001008.

49. William H. Humphries IV, Craig J. Szymanski, and Christine K. Payne. Endo-Lysosomal Vesicles Positive for Rab7 and LAMP1 Are Terminal Vesicles for the Transport of Dextran. PLOS ONE, 6(10):e26626, oct 2011. ISSN 1932-6203. doi: 10.1371/JOURNAL.PONE. 0026626 .

50. Clare E Futter, Adele Pearse, Lindsay J Hewlett, and Colin R Hopkins. Multivesicular endosomes containing internalized EGF-EGF receptor complexes mature and then fuse directly with lysosomes. Journal of Cell Biology, 132(6):1011-1023, 1996. ISSN 00219525. doi: 10.1083/jcb.132.6.1011.

51. Deborah Leonard, Akira Hayakawa, Deirdre Lawe, David Lambright, Karl D. Bellve, Clive Standley, Lawrence M. Lifshitz, Kevin E. Fogarty, and Silvia Corvera. Sorting of EGF and transferrin at the plasma membrane and by cargo-specific signaling to EEA1-enriched endosomes. Journal of Cell Science, 121(20):3445-3458, oct 2008. ISSN 1477-9137. doi: $10.1242 /$ jcs. 031484

52. Martina Trokter, Norbert Mücke, and Thomas Surrey. Reconstitution of the human cytoplasmic dynein complex. PNAS, pages 1-6, 2012. doi: 10.1073/pnas.1210573110.

53. D. M. Gowers, G. G. Wilson, and S. E. Halford. From The Cover: Measurement of the contributions of $1 \mathrm{D}$ and $3 \mathrm{D}$ pathways to the translocation of a protein along DNA. Proceedings of the National Academy of Sciences, 102(44):15883-15888, nov 2005. ISSN 0027-8424. doi: 10.1073/pnas.0505378102.

54. P. Hammar, P. Leroy, A. Mahmutovic, E. G. Marklund, O. G. Berg, and J. Elf. The lac Repressor Displays Facilitated Diffusion in Living Cells. Science, 336(6088):1595-1598, jun 2012. ISSN 0036-8075. doi: 10.1126/science.1221648. 
bioRxiv preprint doi: https://doi.org/10.1101/2021.04.05.438428; this version posted November 1, 2021. The copyright holder for this preprint (which was not certified by peer review) is the author/funder, who has granted bioRxiv a license to display the preprint in perpetuity. It is made available under aCC-BY-NC-ND 4.0 International license.

55. Daniel T. Gillespie. Exact stochastic simulation of coupled chemical reactions. The Journal of Physical Chemistry, 81(25):2340-2361, 1977. doi: 10.1021/j100540a008.

56. Radek Erban and S. Jonathan Chapman. Stochastic Modelling of Reaction-Diffusion Processes. Cambridge Texts in Applied Mathematics. Cambridge University Press, 2020. doi: $10.1017 / 9781108628389$.

57. H.C. Berg, A. Borowski, and E.R. De Vivie. E. coli in Motion. Biological and Medical Physics, Biomedical Engineering. Springer, 2004. ISBN 9780387008882.

58. J. Tailleur and M. Cates. Statistical Mechanics of Interacting Run-and-Tumble Bacteria. Physical Review Letters, 100(21):218103, May 2008. doi: 10.1103/PhysRevLett.100.218103.

59. Clemens Bechinger, Roberto Di Leonardo, Hartmut Löwen, Charles Reichhardt, Giorgio Volpe, and Giovanni Volpe. Active Particles in Complex and Crowded Environments. Reviews of Modern Physics, 88(4):045006, November 2016. doi: 10.1103/RevModPhys.88. 045006.

60. Kanaya Malakar, V. Jemseena, Anupam Kundu, K. Vijay Kumar, Sanjib Sabhapandit, Satya N. Majumdar, S Redner, and Abhishek Dhar. Steady state, relaxation and firstpassage properties of a run-and-tumble particle in one-dimension. Journal of Statistical Mechanics: Theory and Experiment, 2018(4):043215, 2018. doi: 10.1088/1742-5468/aab84f.

61. D. A. Smith and R. M. Simmons. Models of Motor-Assisted Transport of Intracellular Particles. Biophysical Journal, 80(1):45-68, January 2001. doi: 10.1016/S0006-3495(01) 75994-2.

62. Paul Bressloff and Jay Newby. Stochastic models of intracellular transport. Reviews of Modern Physics, 85(1):135-196, January 2013. doi: 10.1103/RevModPhys.85.135.

63. Arthur D Edelstein, Mark A Tsuchida, Nenad Amodaj, Henry Pinkard, Ronald D Vale, and Nico Stuurman. Advanced methods of microscope control using 11/4Manager software. Journal of Biological Methods, 1(2):e10, nov 2014. ISSN 2326-9901. doi: 10.14440/jbm.2014.36.

64. Alexander Krull, André Steinborn, Vaishnavi Ananthanarayanan, Damien RamunnoJohnson, Uwe Petersohn, and Iva M. Tolić-Nørrelykke. A divide and conquer strategy for the maximum likelihood localization of low intensity objects. Optics Express, 22(1):210-228, 2014.

65. Johannes Schindelin, Ignacio Arganda-Carreras, Erwin Frise, Verena Kaynig, Mark Longair, Tobias Pietzsch, Stephan Preibisch, Curtis Rueden, Stephan Saalfeld, Benjamin Schmid, Jean Yves Tinevez, Daniel James White, Volker Hartenstein, Kevin Eliceiri, Pavel Tomancak, and Albert Cardona. Fiji: An open-source platform for biological-image analysis, 2012. ISSN 15487091.

66. Florens Rohde, Ulf Dietrich Braumann, and Matthias Schmidt. Correlia: an ImageJ plug-in to co-register and visualise multimodal correlative micrographs. Journal of Microscopy, 280 (1):3-11, oct 2020. ISSN 13652818. doi: 10.1111/jmi.12928. 